STRUCTURAL SCIENCE CRYSTAL ENGINEERING MATERIALS

ISSN 2052-5206

Received 28 February 2017

Accepted 31 May 2017

Edited by C. Lecomte, Université de Lorraine France

Keywords: spin information transmission; polarized neutron diffraction; spin density; Source Function; spin polarization; spin delocalization; spin density quality.

Supporting information: this article has supporting information at journals.iucr.org/b

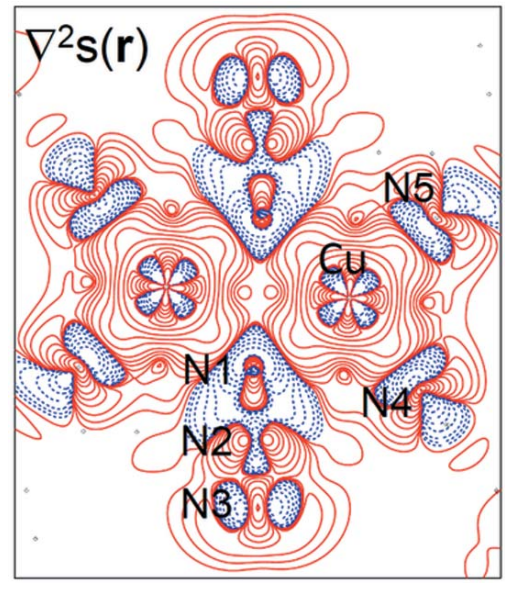

C 2017 International Union of Crystallography

\section{Insights on spin delocalization and spin polarization mechanisms in crystals of azido copper(II) dinuclear complexes through the electron spin density Source Function}

\author{
Carlo Gatti, $^{\text {a* Giovanni Macetti }}{ }^{\mathrm{b}}$ and Leonardo Lo Presti ${ }^{\mathrm{b}}$
}

${ }^{\mathbf{a} C N R}$-ISTM Istituto di Scienze e Tecnologie Molecolari, via Golgi 19, I-20133 Milano, Italy, and ${ }^{\mathbf{b}}$ Department of Chemistry, Università degli Studi di Milano, via Golgi 19, I-20133 Milano, Italy. *Correspondence e-mail: c.gatti@istm.cnr.it

The Source Function (SF) tool was applied to the analysis of the theoretical spin density in azido $\mathrm{Cu}^{\mathrm{II}}$ dinuclear complexes, where the azido group, acting as a coupler between the $\mathrm{Cu}^{\mathrm{II}}$ cations, is linked to the metal centres either in an endon or in an end-end fashion. Results for only the former structural arrangement are reported in the present paper. The SF highlights to which extent the magnetic centres contribute to determine the local spin delocalization and polarization at any point in the dimetallic complex and whether an atom or group of atoms of the ligands act in favour or against a given local spin delocalization/polarization. Ball-and-stick atomic SF percentage representations allow for a visualization of the magnetic pathways and of the specific role played by each atom along these paths, at given reference points. Decomposition of SF contributions in terms of a magnetic and of a relaxation component provides further insight. Reconstruction of partial spin densities by means of the Source Function has for the first time been introduced. At variance with the standard SF percentage representations, such reconstructions offer a simultaneous view of the sources originating from specific subsets of contributing atoms, in a selected molecular plane or in the whole space, and are therefore particularly informative. The SF tool is also used to evaluate the accuracy of the analysed spin densities. It is found that those obtained at the unrestricted B3LYP DFT level, relative to those computed at the $\operatorname{CASSCF}(6,6)$ level, greatly overestimate spin delocalization to the ligands, but comparatively underestimate magnetic connection (spin transmission) among atoms, along the magnetic pathways. As a consequence of its excessive spin delocalization, the UB3LYP method also overestimates spin polarization mechanisms between the paramagnetic centres and the ligands. Spin delocalization measures derived from the refinement of Polarized Neutron Diffraction data seem in general superior to those obtained through the DFT UB3LYP approach and closer to the far more accurate CASSCF results. It is also shown that a visual agreement on the spin-resolved electron densities $\rho_{\alpha}$ and $\rho_{\beta}$ derived from different approaches does not warrant a corresponding agreement between their associated spin densities.

\section{Introduction}

Electron distributions are quantum mechanical observables and scattering techniques enable their model reconstruction in crystalline solids (Coppens, 1997). Focusing on the real space representation of such distributions, X-ray and electron diffraction lead to the crystalline electron position density $\rho(\mathbf{r})$, while its spin counterpart, $s(\mathbf{r})$, may be derived from polarized neutron diffraction (PND) experiments on magnetic crystals (Gillon \& Becker, 2012).

During the last 50 years, Philip Coppens has played a pivotal role in developing techniques to derive increasingly 
accurate X-ray electron densities and in popularizing their use through several landmark papers (for a summary, see Coppens, 2015; Koritsanszky \& Coppens, 2001) and the comprehensive book on $X$-ray Charge Densities and Chemical Bonding (Coppens, 1997). Thanks to him and to many other practitioners, the field could develop so strongly to make him feel confident enough, in 2009, to write a short report in Angewandte Chemie entitled Charge Densities Come of Age (Coppens, 2009). If his view may be essentially shared when the charge density is taken alone, large scope for important developments appears to be still at hand, when considering the synergy of information from the analysis of several electron distributions or by mixing different experimental techniques, experiments and theories, or by developing new interpretive tools. 'The whole is more than the sum of his parts' is the underlying idea in a review article by Macchi et al. (2015) which appeared recently in IUCrJ. That review focused on the possibility 'to extract more information from experimental data, which necessarily means challenging well established models and testing extensions, corrections or even alternative routes' (Macchi et al., 2015). In particular, it recalls that a quite relevant intermediate step has recently been achieved in the long-term quest for a simultaneous refinement of charge, spin and momentum distributions (Gillet et al., 2001; Gillet, 2007 Gillet \& Koritsanszky, 2012). Deutsch et al. (2012, 2014) could in fact for the first time simultaneously derive both the electron position density $\rho(\mathbf{r})$ and its resolved spin components, $\rho_{\alpha}(\mathbf{r})$ and $\rho_{\beta}(\mathbf{r})$ by introducing a spin-split version of the well known Hansen \& Coppens (1978) multipolar model to refine the parameters of a multipolar model against X-ray and PND data. Spin-up and spin-down distributions, which for several reasons cannot be accessed from standard refinements (Deutsch et al., 2012, 2014), were found to be in quite good agreement with those obtained from theoretical computations. In future, such a model extension should lead to increasingly accurate spin density distributions in crystals, $s(\mathbf{r})=\rho_{\alpha}(\mathbf{r})-$ $\rho_{\beta}(\mathbf{r})$, which represent most valuable quantities to visualize the magnetic interaction pathways and their signs and therefore a precious tool to understand and design specific magnetic interactions in complex solid-state networks (Gillon et al., 2007; Deutsch et al., 2012, 2014).

Deciphering the mechanisms through which spin information propagates from paramagnetic to non-magnetic centres and the reasons for possible spin polarization effects requires, however, interpretive models, generally based on atomic or molecular orbital pictures, which are inherently unavailable from experiment and represent just a model anyway. A viable and more rigorous alternative is to resort to descriptors directly based on observables, thus applicable also to observables derived from experiment.

Some time ago, Bader \& Gatti (1998) introduced the concept of the Source Function (SF), which shows that the electron density $\rho(\mathbf{r})$ at any point in the molecular or crystalline space is caused by local source contributions from all other points in the space, $\mathbf{r}^{\prime}$. The SF enables us to visualize the electron-density distribution in terms of cause-effect relationships (Gatti, 2012), being so profoundly connected to 'one of the main operative notions of chemistry, namely that any local property and chemical behaviour of a system is to some extent always influenced by the remaining parts of the system. Whether such an influence is small or large, it is just quantified through the SF' (Gatti, Saleh \& Lo Presti, 2016). Besides investigating whether the SF might also be used to reveal electron delocalization effects in crystals, a recent Acta Crystallographica Section $B$ feature article summarizes what the SF descriptor is and shows a number of its applications using electron densities derived from X-ray diffraction data (Gatti, Saleh \& Lo Presti, 2016). In another recent paper (Gatti et al., 2015), the SF concept has been extended and applied to the electron spin density (SD). Like the standard SF, the electron spin density Source Function provides quantitative insights into the relative capability of different atoms or groups of atoms in a system to determine the spin density at a given system's locations. It does not only show, within a cause-effect view, how spin information propagates from paramagnetic to non-magnetic centres, but whether also these latter may in turn influence the spin density distribution of the paramagnetic centres themselves. And if so, whether an atom or group of atoms concurs or opposes the paramagnetic centres in determining the spin polarization at a given point and whether it does this in a relevant or negligible measure.

Extension of the SF to the reconstruction of the SD was tested on a water molecule in its triplet state (Gatti et al., 2015) and on alkyl radicals (Gatti, Orlando, Monza \& Presti, 2016). Despite the inherent simplicity of these systems, interpretation of the spin density Source Function appears definitely less trivial than the corresponding function for $\rho(\mathbf{r})$. Decomposition of each spin density SF value in a magnetic and in a relaxation (or reaction) term was found not only to ease such an interpretation, providing further very interesting insight (Gatti et al., 2015), but also to be a valid tool to discuss the accuracy of SD distributions. Such an accuracy appears to be a relevant and delicate issue when comparing SD distributions obtained from model wavefunctions of different quality (Boguslawski et al., 2011, 2012; Gatti et al., 2015) or when contrasting experimental and theoretical SD distributions.

As a follow-up to our previous studies, we have applied this novel SF descriptor to the theoretical SD distribution of two azido $\mathrm{Cu}^{\mathrm{II}}$ complexes, using molecular structures taken from their molecular crystals (Aebersold et al., 1998; Aronica et al., 2007). For limits of space, only the results for the end-on complex can be reported in this paper. Those for the end-end complex, along with a comparison between the SF spin density descriptions of the two complexes, will be reported in future work. Our work has several purposes. Firstly, our approach needs to be tested on more complex systems than carried out thus far (Gatti et al., 2015; Gatti, Orlando, Monza \& Lo Presti, 2016). Secondly, the kind of information that may be retrieved from a spin density distribution using a descriptor that potentially also applies to experimentally derived spin densities requires further exploration. Indeed, the spin densities of the two compounds we have investigated have in the past also been obtained from standard multipolar model refinements and theoretical computations (Aebersold et al., 1998; Aronica 
et al., 2007) and, for one system, also through simultaneous Xray and PND data refinement (Deutsch et al., 2014). Other purposes of this paper concern an important methodological improvement, related to the problem of easing the choice of the reference points in the SF analysis and to the desire to further assess the capability of our approach to physically analyse and quantify spin density changes as a function of the SD distribution quality.

The paper is organized as follows. §2 reviews the Source Function for the spin density and introduces the concept of partial spin density SF reconstructions due to a given subset of atoms in the system. $\$ 3$ succinctly describes the two analysed magnetic molecules and their geometrical and magnetostructural features, while $\$ 4$ reports some computational details. §5 illustrates the SF view of the spin delocalization and polarization mechanisms in the end-on azido $\mathrm{Cu}^{\mathrm{II}}$ dinuclear complex. §6 outlines the Conclusions.

\section{The Source Function descriptor}

\subsection{The Source Function for the electron density}

The electron density at any point $\mathbf{r}$ of a closed quantum system with boundary at infinity is caused by a Local Source (LS), LS(r, $\left.\mathbf{r}^{\prime}\right)$, which operates at all other points $\mathbf{r}^{\prime}$ of the system

$$
\rho(\mathbf{r})=\int \operatorname{LS}\left(\mathbf{r}, \mathbf{r}^{\prime}\right) \mathrm{d} \mathbf{r}^{\prime}
$$

and which depends on their electron-density Laplacian values

$$
\operatorname{LS}\left(\mathbf{r}, \mathbf{r}^{\prime}\right)=-\left(4 \pi \cdot\left|\mathbf{r}-\mathbf{r}^{\prime}\right|\right)^{-1} \cdot \nabla^{2} \rho\left(\mathbf{r}^{\prime}\right) .
$$

The factor $\left(4 \pi \cdot\left|\mathbf{r}-\mathbf{r}^{\prime}\right|\right)^{-1}$ is a Green's function, that is the influence function (Arfken, 1985) characterizing how effective is the cause, $\nabla^{2} \rho\left(\mathbf{r}^{\prime}\right)$, in determining the effect, $\rho(\mathbf{r})$. The whole space integration of the LS may then be conveniently replaced (Bader \& Gatti, 1998) by separate integrations over the atomic basins defined through the disjoint and exhaustive space partitioning provided by the surface boundary condition of local null zero-flux of the $\nabla \rho(\mathbf{r})$ vector field, i.e. the socalled zero-flux surface recipe of the quantum theory of atoms in molecules, i.e. QTAIM (Bader, 1990). Operating in such a way, $\rho(\mathbf{r})$ can be decomposed in terms of the sum of the atomic basin SF contributions

$$
\rho(\mathbf{r})=S(\mathbf{r}, \Omega)+\sum_{\Omega^{\prime} \neq \Omega} S\left(\mathbf{r}, \Omega^{\prime}\right),
$$

where distinction has been made in the sum between the source from the atomic basin hosting the point where the density is reconstructed, the so-called reference point $r p$, and the cumulative sources from the remaining basins $\Omega^{\prime}$. Partitioning of space according to the QTAIM recipe ensures a quantum mechanical rigorous association of the sources in equation (3) to the atoms or group of atoms ('functional groups') of the system, though any mutually exclusive or fuzzy space partitioning scheme could in principle also be adopted (Gatti, 2012). Obviously, rigorous association also implies chemical insight since the value of the electron density at a point and its change upon any perturbation caused by chemical substitution, change of geometry, change of environment, etc., may then be interpreted in terms of how the atoms in a system contribute to determine both of them. By requiring only knowledge of the electron density and of its Laplacian, the SF descriptor is experimentally accessible from X-ray diffraction intensity data through multipole model (MM) refinement (Hansen \& Coppens, 1978; Stewart et al., 1975; Volkov et al., 2006; Gatti \& Macchi, 2012).

\subsection{The Source Function for the spin density}

Extension of the SF to the SD case is mathematically straightforward (Gatti et al., 2015). One just needs to replace $\rho(\mathbf{r})$ by $s(\mathbf{r})$, both in the local cause, $\nabla^{2} \rho\left(\mathbf{r}^{\prime}\right) \rightarrow \nabla^{2} s\left(\mathbf{r}^{\prime}\right)$, and in the effect, $\rho(\mathbf{r}) \rightarrow s(\mathbf{r})$, while the Green function, $\left(4 \pi\left|\mathbf{r}-\mathbf{r}^{\prime}\right|\right)^{-1}$, being a purely geometrical (effectiveness) factor, independent from the scalar to be reconstructed, remains unaltered. The symmetry of the $\rho \rightarrow s$ scalar replacement is broken for what concerns the space partitioning in atomic basins. Such a partitioning is, also for the SD, performed using the $\nabla \rho(\mathbf{r})$ zero-flux QTAIM recipe to maintain the rigorous association of atomic source contributions with the quantum atoms or group of atoms of Bader's theory.

The SF decomposition for $s(\mathbf{r})$ will then be expressed as

$$
s(\mathbf{r})=\int_{R^{3}} \operatorname{LS}_{\mathrm{S}}\left(\mathbf{r}, \mathbf{r}^{\prime}\right) \mathrm{d} \mathbf{r}^{\prime}=\sum_{\Omega} \int_{\Omega} \operatorname{LS}_{\mathrm{S}}\left(\mathbf{r}, \mathbf{r}^{\prime}\right) \mathrm{d} \mathbf{r}^{\prime}=\sum_{\Omega} S_{\mathrm{s}}(\mathbf{r}, \Omega),
$$

where $S_{\mathrm{S}}(\mathbf{r}, \Omega)$ is the SF contribution from atom $\Omega$ to $s(\mathbf{r})$ and the local source for the spin density, $\mathrm{LS}_{\mathrm{S}}$, assumes the form

$$
\begin{aligned}
\operatorname{LS}_{\mathrm{S}}\left(\mathbf{r}, \mathbf{r}^{\prime}\right) & =-\frac{1}{4 \pi} \frac{\nabla^{2} s\left(\mathbf{r}^{\prime}\right)}{\left|\mathbf{r}-\mathbf{r}^{\prime}\right|}=-\frac{\nabla^{2}\left[\rho_{\alpha}\left(\mathbf{r}^{\prime}\right)-\rho_{\beta}\left(\mathbf{r}^{\prime}\right)\right]}{4 \pi\left|\mathbf{r}-\mathbf{r}^{\prime}\right|} \\
& =\frac{\nabla^{2} \rho_{\beta}\left(\mathbf{r}^{\prime}\right)-\nabla^{2} \rho_{\alpha}\left(\mathbf{r}^{\prime}\right)}{4 \pi\left|\mathbf{r}-\mathbf{r}^{\prime}\right|} .
\end{aligned}
$$

Replacement of $\nabla^{2} \rho\left(\mathbf{r}^{\prime}\right)$ by $\nabla^{2} s\left(\mathbf{r}^{\prime}\right)$ in the local source expression generally leads to quite different atomic sources for the reconstruction of the $\rho(\mathbf{r})$ and $s(\mathbf{r})$ scalars at a given reference point $\mathbf{r}$ (Gatti et al., 2015). In fact, the two scalar fields were generally found to concentrate $\left[\nabla^{2} u\left(\mathbf{r}^{\prime}\right)<0, u=s\right.$ or $\rho$ ] or dilute $\left[\nabla^{2} u\left(\mathbf{r}^{\prime}\right)>0\right]$ themselves in a quite distinct manner, implying that the electron density and the SD information transmit themselves with largely different mechanisms throughout a system (Gatti et al., 2015; Gatti, Orlando, Monza $\&$ Lo Presti, 2016). The fact that $s(\mathbf{r})$ is given by the difference of the $\alpha$ - and $\beta$-electron-density components then yields further peculiarities. Concentration or dilution of both density components is not sufficient to ensure a positive and a negative local source contribution, respectively. What matters is the relative concentration or dilution of the two components (Gatti et al., 2015). For instance, when both $\alpha$ - and $\beta$-distributions are concentrated, the local source $\mathrm{LS}_{\mathrm{S}}$ will be positive if $\left|\nabla^{2} \rho_{\alpha}\left(\mathbf{r}^{\prime}\right)\right|>\left|\nabla^{2} \rho_{\beta}\left(\mathbf{r}^{\prime}\right)\right|$, while it will be negative if it is the $\beta$ density which is more concentrated than the $\alpha$-density, $\left(\left|\nabla^{2} \rho_{\beta}\left(\mathbf{r}^{\prime}\right)\right|>\left|\nabla^{2} \rho_{\alpha}\left(\mathbf{r}^{\prime}\right)\right|\right)$. Conversely, when both distributions are diluted, the local source $L S_{S}$ will be positive if $\nabla^{2} \rho_{\beta}\left(\mathbf{r}^{\prime}\right)>$ 
$\nabla^{2} \rho_{\alpha}\left(\mathbf{r}^{\prime}\right)$ and it will be negative if it is the $\alpha$-density which is more locally diluted, $\nabla^{2} \rho_{\alpha}\left(\mathbf{r}^{\prime}\right)>\nabla^{2} \rho_{\beta}\left(\mathbf{r}^{\prime}\right)$. The list of all possible cases, including the situations in which one component is concentrated and the other is diluted, is detailed in Table 2 of Gatti et al. (2015). A positive local source $\mathrm{LS}_{\mathrm{S}}$ [i.e. $\left.\nabla^{2} s\left(\mathbf{r}^{\prime}\right)<0\right]$ will cause an increase of the $\alpha$-component of the total electron density, i.e. of its $\alpha$-spin polarization, at a given $r p \mathbf{r}$. Such an increase has been named an $\alpha$ effect, while a $\beta$ effect (Gatti et al., 2015) is the increase of the $\beta$-component at $\mathbf{r}$ caused by a negative local source $\operatorname{LS}_{\mathrm{S}}\left[\right.$ i.e. $\left.\nabla^{2} s\left(\mathbf{r}^{\prime}\right)>0\right]$. The $\alpha$ or $\beta$ nature of the effect is only a function of the source point $\mathbf{r}^{\prime}$, while its magnitude, besides the spin Laplacian magnitude, is due to the distance from the $r p$.

As anticipated in $\$ 1$, interpretation of $S_{\mathrm{S}}$ values is not straightforward and it is largely eased and deepened when they are split into a magnetic and a reaction (or relaxation) contribution (Gatti et al., 2015). The former contribution is defined as that due to the distribution of the fully unpaired $\alpha$ electrons, while the latter is defined as that originating from the distribution of the remaining $\alpha$ and $\beta$ electrons. The magnetic density integrates, over the whole space, to the number of unpaired electrons in the system, while the reaction or relaxation density has an associated SD which integrates to zero over such space, but may, in general, be locally different from zero. It is called reaction or relaxation density as the $\int \rho_{\alpha} \equiv \int \rho_{\beta}$ overall constraint is locally relaxed $\left(\rho_{\alpha} \neq \rho_{\beta}\right)$ as a reaction to the presence of the distribution of the unpaired electrons. It is worth noting that the magnetic $\mathrm{LS}_{\mathrm{S}}$, though being due only to $\alpha$-electron density, may have either an $\alpha$ or a $\beta$ effect, depending on the sign of its Laplacian, while the $\mathrm{LS}_{\mathrm{S}}$ due to the reaction density may either counteract or enhance the $\alpha$ or $\beta$ effect due to the magnetic density. The magnetic density is easily obtained by diagonalizing the first-order density matrix and by selecting, in the evaluation of the density, only those natural orbitals having occupations equal to or very close to one. A thorough discussion and technical details on this mentioned density decomposition is given in Gatti et al. (2015), while further technical details relevant to the present work are highlighted in the computational details presented in $\S 4$.

\subsection{Representations of the Source Function descriptor and selection of the reference points}

Atomic SF contributions for the electron density may be analysed as such or more often in terms of Source Function percentage contributions, $S \%$

$$
S \%(r p, \Omega)=\frac{S(r p, \Omega)}{\rho(r p)} \times 100,
$$

which express the relative capability of an atom or group of atoms $\Omega$ to determine the electron density at the $r p$ (Gatti, 2012). Likewise, Source Function percentages for the SD, $S_{\mathrm{S}} \%$, will be given by

$$
S_{\mathrm{S}} \%(r p, \Omega)=\frac{S_{\mathrm{S}}(r p, \Omega)}{s(r p)} \times 100 .
$$

Regardless of using absolute or percentage SF contributions, the proper choice, if any, of the reference points is one obvious issue to be faced when the SF descriptor is applied. Which guiding criteria should be followed in selecting the rps? Also, once chosen, how can one judge whether the obtained atomic SF patterns are representative or not of a given molecular or crystal region? In other words, why those rps and not any other?

When dealing with the investigation of chemical bond features, it looks reasonable to take the electron-density bond critical points (BCPs; Bader, 1990) as the most representative and least biased choices for $r p$ s associated with a bonding interaction (Gatti et al., 2003; Gatti, 2012). There may also be the case where significant electron sharing exists between two atoms despite the fact that they are not connected by a bond path (Pendás et al., 2007; Farrugia et al., 2006; Gatti \& Lasi, 2007; Ponec \& Gatti, 2009; Gatti, 2013). In this event, the evaluation of SF contributions at the internuclear axis midpoint and along the whole axis was found to be appropriate (Farrugia et al., 2006; Gatti \& Lasi, 2007; Gatti, 2012). However, the most suited choice of $r p$ s becomes less evident when considering the electron spin density SF reconstructions. For instance, it has been found that the SD is often minimized at BCPs, because at these positions, particularly for covalent bonds, electron pairing is maximized and the magnetic density only plays a minor role here (Gatti et al., 2015; Gatti, Orlando, Monza \& Lo Presti, 2016). Representative points for such density, which largely determines the SD in the high $\alpha$-polarization density regions, have been taken at the critical points of the electron-density Laplacian distribution associated with unpaired electrons and, for the sake of comparison, also at the $(3,-3)-\nabla^{2} \rho$ critical points associated with lone pair electrons (Gatti et al., 2015). However, such choices are somewhat arbitrary and possibly biased. An obvious solution, which is presented here for the first time, is to calculate the SF contributions from a given subset of the atoms in the system at an $N$-dimensional grid of $r p s$. In this way, cube $(N=3)$, surface $(N=2)$, line $(N=1)$ or point $(N=0)$ electron or electron spin partially reconstructed densities are obtained

$$
\begin{gathered}
\rho\{\Omega, \text { subset }\}(\mathbf{r})=\sum_{\Omega, \text { subset }} \int \operatorname{LS}\left(\mathbf{r}, \mathbf{r}^{\prime}\right) \mathrm{d} \mathbf{r}^{\prime}=\sum_{\Omega, \text { subset }} S(\mathbf{r}, \Omega) \\
s\{\Omega, \text { subset }\}(\mathbf{r})=\sum_{\Omega, \text { subset }} \int \operatorname{LS}_{\mathrm{s}}\left(\mathbf{r}, \mathbf{r}^{\prime}\right) \mathrm{d} \mathbf{r}^{\prime}=\sum_{\Omega, \text { subset }} S_{\mathrm{s}}(\mathbf{r}, \Omega) .
\end{gathered}
$$

When the subset includes all the atoms of the system, these reconstructed densities are no longer partial. Their deviations from the corresponding densities are usually negligible and provide just a measure of the numerical quality of the reconstruction through the SF descriptor. When, instead, only an atom or a subset of the atoms of the system is considered in equation (8) or (9), the role this atom or group of atoms has in determining either the electron or the SD in a given region of space becomes manifest. Note that there is a profound difference between the SF partially reconstructed densities and those partial density representations one may obtain using 
a subset of pseudoatom densities in the multipolar model approaches (Volkov et al., 2006) or using only the contributions from the basis functions of a subset of atoms in a theoretical computation. The SF partially reconstructed densities yield a cause-effect picture of atomic contributions in determining a given density and in this respect are fully unbiased. The information they provide is only a function of the total density they partially reconstruct. The standard partial densities, instead, refer to actual density contributions from pseudoatom monopolar density functions or from atomic basis set functions and depend, not only on the total density, but also on the specific multipole model or atomic basis set adopted. At the limit, if a complete basis set centred on a point other than a nuclear position were used in a theoretical computation, the resulting electron or spin density would be the best which could be obtained within the chosen wavefunction expression, yet all partially reconstructed densities would be equal to zero everywhere.

Partially reconstructed densities may be portrayed as such or as percentage reconstructed densities. When $N=0$, percentage sources are typically shown in a ball-and-stick representation for all atoms in a system (or at least for those atoms yielding significant sources). Each atom is assigned a volume proportional to its SF percentage contribution and such a representation pictorially illustrates whether the reconstruction of the density at the $r p$ is made in terms of very localized or in terms of partially or totally delocalized sources. On the other hand, when compared with the total density, the two-dimensional contour maps of the partially reconstructed densities give a very clear indication of how an atom or a subset of atoms contribute to determine the electron or the SD in the selected plane. These maps thus depict whether in a given molecular region such a contribution is important, modest or negligible and whether it remains almost stable or significantly varies within the region. These maps may therefore be quite useful for a proper selection of the reference point for an $N=0$ representation and for a full understanding and relevance assessment of such a representation.

As a summarizing example, Fig. 1 illustrates a number of representations of the electron spin density SF contributions (Gatti et al., 2015) in the triplet ${ }^{3} \mathrm{~B}_{1} \mathrm{H}_{2} \mathrm{O}$ system (Herzberg, 1966) computed at the $\operatorname{CASSCF}(8,8)$ level and using a 6$311++\mathrm{G}(2 \mathrm{~d}, 2 \mathrm{p})$ basis set. In the first row of the figure, a balland-stick $N=0$ representation of the atomic SF percentage contributions at the $\mathrm{O}-\mathrm{H} \mathrm{BCP}(s=-0.008$ a.u. $)$ is shown for the total SD and for its magnetic and relaxation components. Green (red) balls denote an $\alpha(\beta)$ effect on the density at this $r p$, while SF percentage contributions are taken as positive or negative, whether the sources have the same or the opposite sign of the density value at the $r p$. It is clear that in the molecular plane there is a spin polarization mechanism, since the $\mathrm{H}$ atoms determine positive SD contributions at the BCP, and hence have an $\alpha$ effect, while the $\mathrm{O}$ atom behaves in the opposite manner and its dominant $\beta$ effect leads to a negative $\mathrm{SD}$ at the BCP (Gatti et al., 2015). Surprisingly, $\mathrm{H}$ and $\mathrm{H}^{\prime}$ atoms yield very similar sources $(-29.1$ and $-25.5 \%$, respectively), despite the $r p$ relating only $\mathrm{O}$ and $\mathrm{H}$ directly, suggesting that some compensating mechanism is operative here. The relaxation term, which yields a $\beta$ effect for all atoms, neatly differentiates the effects of $\mathrm{H}$ and $\mathrm{H}^{\prime}$, similar to what is found when the electron density is reconstructed (Gatti et al., 2015). It is the dominant and, as far as the $\mathrm{H}$ atoms are concerned, opposing magnetic term that eventually leads to similar total sources from the two $\mathrm{H}$ atoms. Due to spin delocalization and polarization effects, the magnetic source from $\mathrm{H}$ is only four times larger than that of $\mathrm{H}^{\prime}(-117.8$ versus $-31.4 \%$ ), while the corresponding relaxation sources, which are related to a through-bond covalent mechanism and are therefore more local in nature, differ by a factor almost four

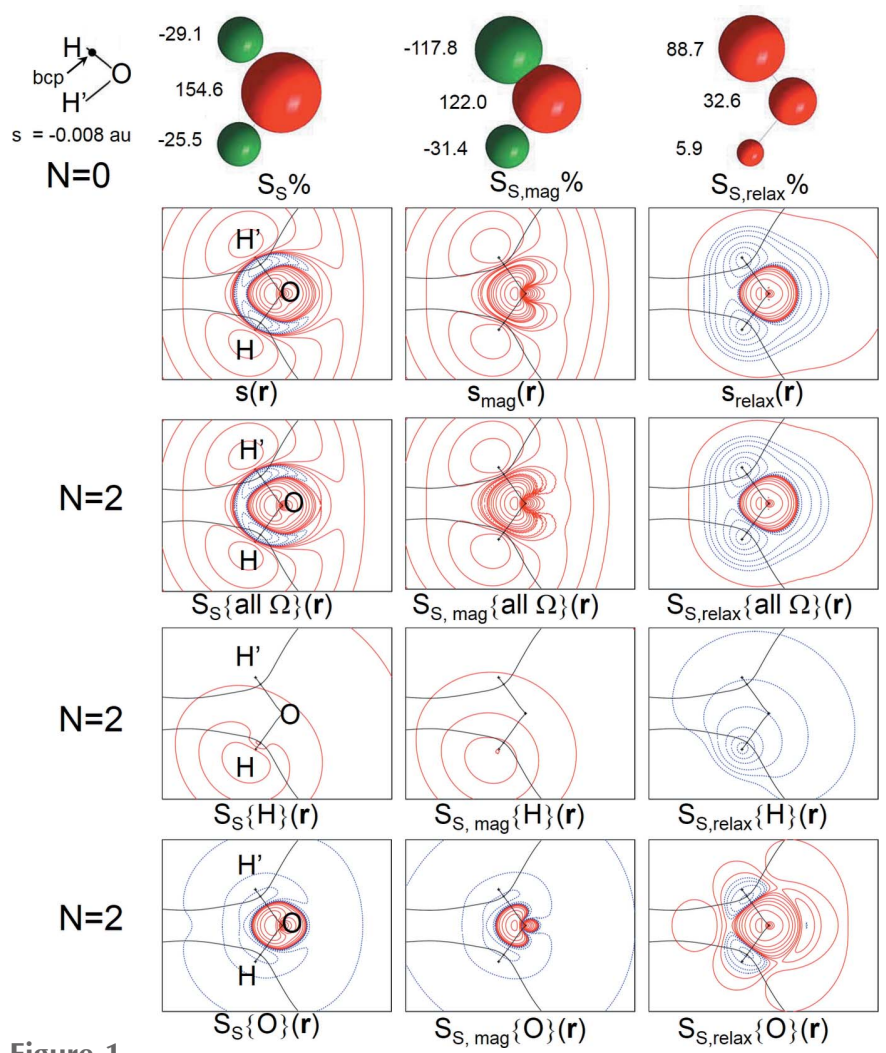

Figure 1

$\mathrm{N}$-dimensional representations of the electron spin density (SD) Source Function contributions for triplet ${ }^{3} \mathrm{~B}_{1} \mathrm{H}_{2} \mathrm{O}$ at the CASSCF $(8,8)$ level and using a $6-311++\mathrm{G}(2 \mathrm{~d}, 2 \mathrm{p})$ basis set. Source Function contributions, electron SD, and total and SF partially reconstructed densities are shown for the SD and for its magnetic and relaxation components. First row: SF percentage contributions for a reference point taken at the $\mathrm{O}-\mathrm{H}$ bond critical point. Green (red) atomic balls denote an $\alpha(\beta)$ effect on the density at the reference point. SF percentage contributions are positive or negative whether they concur or oppose to reconstruct the density value at the reference point. Second row panels: two-dimensional contour plots of the total SD and of its components in the molecular plane [red are positive and dotted blue are negative contour values; contour maps are drawn at an interval of $\pm(2,4,8) \times 10^{n},-4 \leq n \leq 0$ atomic units (a.u.)]. Full black lines mark boundaries of atomic basins. The same conventions and isovalue contour lines occur in all maps of the figure. Third row panels: SF reconstructed densities in the molecular plane, using the sources of all atoms in the molecule. Fourth row panels: as in the third row, but excluding the $\mathrm{H}^{\prime}$ and $\mathrm{O}$ sources. Fifth row panels: as in the third row, but excluding the $\mathrm{H}$ and $\mathrm{H}^{\prime}$ sources. The first row illustrates an $N=0$ (zero-dimensional) SF\% representation, while the panels in the other rows are $N=2$ (two-dimensional) SF representations. [SF AND SF\% OK] 
times as large. The quite different decay law with distance from the $r p$ of the (here opposing) magnetic and relaxation $\mathrm{H}$ atoms sources is the ultimate reason for their similar total sources at the $\mathrm{O}-\mathrm{H}$ BCP. The second row panels in Fig. 1 display contour plots for the SD and its magnetic and relaxation components in the molecular plane. SF reconstructions of such densities using all the atoms of the molecule (third row panels, Fig. 1) lead to essentially identical plots, indicating that the SF numerical reconstruction is almost perfect in this case. The negative SD regions are due to the relaxation spin density, since the magnetic SD of all atoms is positive everywhere, as it must be by definition. The spin polarization mechanism is clearly evident along the bond paths and in significant parts of the O-atom basin. More insight is obtained when all the atom reconstructions are compared against the $N=2$ SF partial SD reconstructions. Those excluding $\mathrm{H}^{\prime}$ and $\mathrm{O}$ atoms are shown in the fourth row panels and those excluding $\mathrm{H}$ and $\mathrm{H}^{\prime}$ atoms in the fifth row panels. From an inspection of the left panels it is evident that only the $\mathrm{O}$ atom is responsible for the regions of negative $\mathrm{SD}$ in the molecular plane ( $\beta$ effect). In such a plane, its $\alpha$ effect is limited to a region close to its nucleus, while each $\mathrm{H}$ atom yields only an $\alpha$ effect everywhere. For $\mathrm{H}$ atoms, the magnetic density everywhere is positive and dominating over the negative relaxation density. The magnetic density due to the $\mathrm{H}$ atoms also prevails over the negative magnetic density brought in by the oxygen in some regions of the molecular plane, thus ensuring the magnetic density to be positive everywhere, as required. The $\mathrm{O}$ atom has a region of reinforcing positive magnetic and relaxation SDs around the nucleus, but also displays regions where these densities are both negative (along the bond path and close to the BCPs and the hydrogen nuclei) or, instead, oppose each other, as in the case of the $\mathrm{H}$ atoms.

\section{Structural and magnetostructural aspects of the investigated azido-bridged dicopper complexes}

Azido-bridged polymetallic species have been largely synthesized and studied since the 1980s for their structural diversity and their interesting and challenging magneto-structural relationships (Aronica et al., 2007, and references therein). The azido group, i.e. $\mathrm{N}_{3}^{-}$, is a non-innocent ligand and one of the most interesting magnetic couplers in molecular magnetism (Aebersold et al., 1998, and references therein). We have performed an SF analysis of the SD in two azido-bridged dicopper molecular complexes, namely $\left[\mathrm{Cu}_{2}(t-\right.$ Bupy $\left.)_{4}\left(\mathrm{~N}_{3}\right)_{2}\right]\left(\mathrm{ClO}_{4}\right)_{2} \quad[t$-Bupy $=p$-tert-butylpyridine $]$ and $\mathrm{Cu}_{2} L_{2}\left(\mathrm{~N}_{3}\right)_{2}$ ( $L=$ 7-dimethylamino-1,1,1-trifluoro-4-methyl-5azahept-3-en-2-onato), whose geometries have been taken from their molecular crystal structures, using the 18 (Aebersold et al., 1998) and $150 \mathrm{~K}$ (Aronica et al., 2007) X-ray geometries, respectively. The $\mathrm{Cu}^{\mathrm{II}}$ cations in the complexes are coupled through two azido bridges; in the former complex, the azido groups are symmetrically linked to the $\mathrm{Cu}^{\mathrm{II}}$ cations through one terminal $\mathrm{N}$ atom $(\mu-1,1)$ (end-on, EO coordination mode), resulting in two short equivalent $\mathrm{N}-\mathrm{Cu}$ bonds. In the second complex, two terminal $\mathrm{N}$ atoms $(\mu-1,3)$ (end-to-end, EE coordination mode) coordinate asymmetrically, i.e. with one short and one long $\mathrm{N}-\mathrm{Cu}$ bond, the two metal centres. Fig. 2 summarizes the relevant structural data. Both complexes are centrosymmetric, with $\mathrm{Cu}^{\prime} \ldots \mathrm{Cu}$ distances as large as $\simeq 3$ (EO) and $5 \AA$ (EE), and ions ferromagnetically coupled to a different extent $\left[J(\mathrm{EO})=300 \mathrm{~cm}^{-1}\right.$ and $\left.J(\mathrm{EE})=17 \mathrm{~cm}^{-1}\right]$ (Aebersold et al., 1998; Aronica et al., 2007). Symmetrically linked azido groups lead to $\mathrm{Cu}^{\prime} \cdots \mathrm{Cu}$ intramolecular magnetic coupling which varies from strongly ferromagnetic to strongly antiferromagnetic, depending on the coordination mode (Aebersold et al., 1998). The EO complexes exhibit a triplet ground state (ferromagnetic, FM, coupling) because of the quasi-orthogonality of the $\mathrm{Cu}^{\mathrm{II}}$ magnetic orbitals for the range of observed values of the $\mathrm{Cu}^{\prime}-\mathrm{N}-\mathrm{Cu}$ bridging angle, while the EE complexes always show a singlet spin state (antiferromagnetic, AF, coupling) due to the strong overlap between these orbitals (Aronica et al., 2007). For asymmetrically bridged complexes, the picture is less definite (Aronica et al., 2007). EO compounds may show AF coupling, while EE complexes may exhibit FM coupling, as in the present case, or, more commonly, an AF coupling. Regardless of the AF or FM nature, coupling in the EE asymmetric systems is, however,

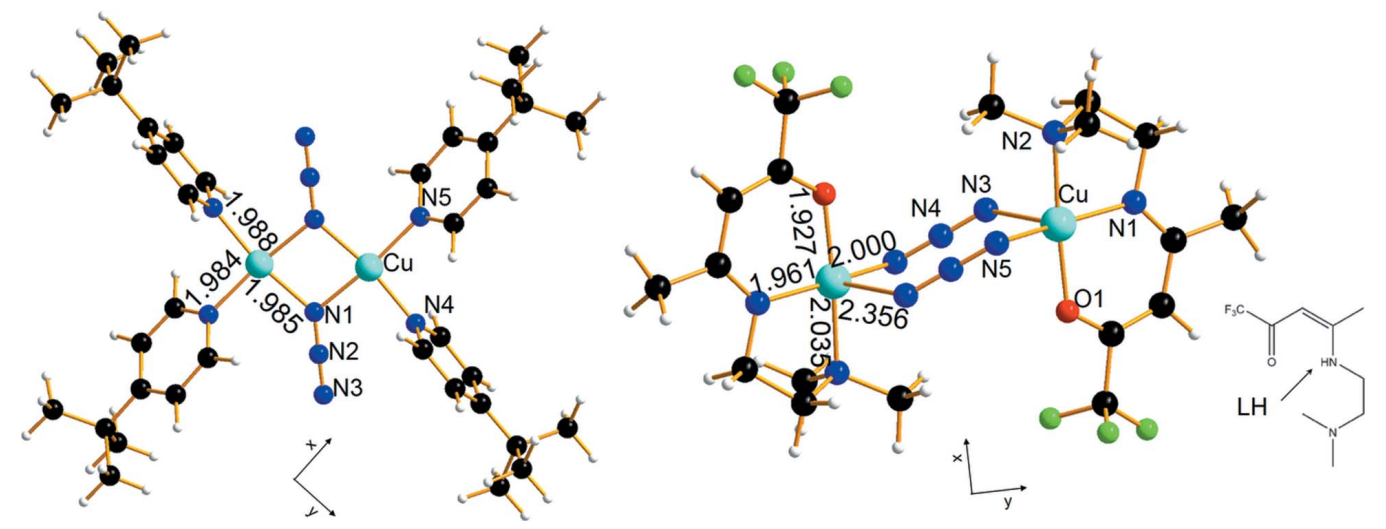

Figure 2

Molecular structure, atomic labelling and geometrical data for the FM end-on (left) and the FM end-end (right) centrosymmetric azido-bridged dicopper molecular complexes. Bond distances are in $\AA$. Black, white and green balls denote $\mathrm{C}, \mathrm{H}$ and $\mathrm{F}$ atoms, respectively. 
Table 1

Net charges $(q)$, spin populations (SP) and atomic spin density Laplacian (ASDL) for selected atoms or atom groups $\Omega$ in the end-on FM azido Cu ${ }^{\mathrm{II}}$ dinuclear complex.

QTAIM partitioning: $q(\Omega)$ and $\mathrm{SP}(\Omega)$; Mulliken's partitioning: $\operatorname{SP}_{\mathrm{M}}(\Omega)$; polarized neutron diffraction estimate: $\mathrm{SP}_{\mathrm{PND}}(\Omega) ; \mathrm{ASDL}_{\Omega} \int \nabla_{\Omega}^{2} s$ dr.

\begin{tabular}{|c|c|c|c|c|c|c|}
\hline$\Omega$ & $q(\Omega) \dagger$ & $\mathrm{SP}(\Omega) \dagger$ & $\operatorname{SP}_{\mathrm{M}}(\Omega) \dagger$ & $\operatorname{SP}_{\mathrm{PND}}(\Omega) \ddagger$ & $\begin{array}{l}\mathrm{SP}_{\mathrm{M}}(\Omega) \ddagger \S \\
\text { (Abersold simplified } \\
\text { structural model) }\end{array}$ & ASDL $\dagger$ \\
\hline $\mathrm{Cu}$ & $1.433(1.095)$ & $0.899(0.619)$ & $0.919(0.628)$ & $0.783(7)$ & 0.425 & $-0.206(-0.085)$ \\
\hline $\mathrm{N} 2$ & $-0.216(-0.138)$ & $-0.009(0.005)$ & $-0.013(-0.007)$ & $-0.016(6)$ & -0.005 & $0.025(0.064)$ \\
\hline N3 & $0.126(+0.039)$ & $0.028(0.088)$ & $0.030(0.100)$ & $0.057(7)$ & 0.122 & $-0.018(-0.047)$ \\
\hline Azide & $-0.733(-0.587)$ & $0.051(0.188)$ & $0.041(0.182)$ & $0.110(19)$ & 0.284 & $0.105(0.053)$ \\
\hline$\sum \mathrm{C}_{\mathrm{py}}(\mathrm{N} 5)$ & $1.264(0.914)$ & $0.002(0.008)$ & $0.002(0.004)$ & $0.005(52)$ & 0.008 & - \\
\hline
\end{tabular}

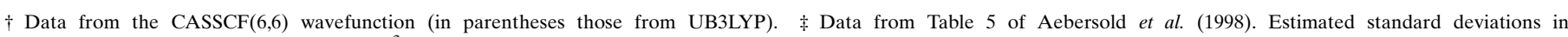

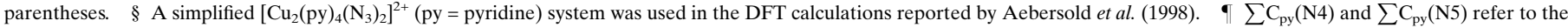
sum of the properties of the $\mathrm{C}$ atoms in the pyridine rings having $\mathrm{N} 4$ or $\mathrm{N} 5$ has their $\mathrm{N}$ atom.

always very low. In fact, in a square-pyramidal geometry coordination of $\mathrm{Cu}^{\mathrm{II}}$ (Fig. 2), one terminal azido $\mathrm{N}$ atom points to the $d_{x^{2}-y^{2}}$ magnetic orbital of one $\mathrm{Cu}^{\mathrm{II}}$ cation, forming the short $\mathrm{Cu}-\mathrm{N}$ bond, while the other points to the $d_{z^{2}}$ orbital of the second $\mathrm{Cu}^{\mathrm{II}}$ cation, forming the long $\mathrm{Cu}^{\prime}-\mathrm{N}$ bond and implying a weak or negligible interaction. As stated before, only results for the EO symmetric complex will be reported in the following. Due to space constraints, the discussion on the relationships between the different structural arrangements around the $\mathrm{Cu}^{\mathrm{II}}$ cation, the strong or weak $\mathrm{Cu}^{\prime} \cdots \mathrm{Cu}$ FM couplings and the different SF spin density portraits in the EO and EE complexes will be reported in future work.

\section{Computational details}

Theoretical SD computations for the triplet ground states of the EO azido-bridged dicopper molecular complex were performed in vacuo and at its crystalline geometry by means of the GAUSSIAN09 program package (Frisch et al., 2009). Both the double positively charged and the neutral complexes were calculated, but results are discussed only for the former, as their spin distributions were found to be almost indistinguishable. Complete active space self-consistent field (CASSCF), UB3LYP (unrestricted B3LYP) and UHF (unrestricted Hartree-Fock) levels of theory were adopted for both complexes, employing the pob-TZVP basis set (Peintinger et al., 2013) for all methods. The reported UB3LYP results refer to spin-contamination annihilated wavefunctions. Spin contamination by states of higher multiplicity than the triplet state was minor $\left[\left\langle S^{2}\right\rangle=2.0109\right]$ and almost zero after the annihilation procedure $\left[\left\langle S^{2}\right\rangle=2.0001\right]$. CASSCF computations included six electrons in the active space composed of six orbitals [CASSCF $(6,6)]$, i.e. four occupied and two virtuals in the initial guess determinant. The starting orbital guess for the CASSCF computation was taken from the UB3LYP spincontamination annihilated natural orbitals. The active orbitals were chosen in an analogous manner to those adopted in the model by Aebersold et al. (1998), selecting both gerade and ungerade symmetries. The UHF method gave serious selfconsistent field convergence problems and high spin contamination of the resulting wavefunction, even after spincontamination annihilation. UHF results are therefore not discussed in the paper, although we checked that the CASSCF converged spin density was practically the same, whether the natural orbitals from the UHF or from the UB3LYP calculations were used as a starting guess. The Slater determinant expansion of the $\operatorname{CASSCF}(6,6)$ wavefunction includes 225 configurations of the correct symmetry and spin multiplicity. The magnetic density of the UB3LYP and CASSCF wavefunctions was calculated from their natural orbitals obtained through the 'pop = NO' option and by selecting only those with occupation numbers equal to one or marginally different from one (highest deviation from one being 0.00029). Spin densities for the various wavefunctions were obtained following the same sequence of intermediate steps detailed in the supplementary information of Gatti et al. (2015).

Topological analysis of the $\rho$ and $\nabla^{2} \rho$ scalar fields was performed through a modified version of the AIMPAC program package (Biegler-König et al., 1982). Other homedeveloped codes have been used to evaluate: (a) atomic Source Function contributions to the electron density, the spin density and its magnetic and relaxation components at selected reference points or at the points of an $N(N=1-3)$ dimensional grid (SPINSF2016 code); (b) spin density and spin density Laplacian, also resolved in their $\alpha$ and $\beta$ contributions at given positions (EXTREMESPIN code); (c) contour two-dimensional maps of the spin density, of the spin density Laplacian and of their magnetic and relaxation densities counterparts; $(d)$ contour two-dimensional maps of the SF reconstructed partial spin densities and their magnetic and relaxation density counterparts (PLOTDEN2016 code). SPINSF2016 and EXTREMESPIN codes are heavily modified versions of the original PROAIMV and EXTREME codes of the AIMPAC program package, while PLOTDEN2016 is an updated and unpublished version of the PLOTDEN2013 code (also unpublished, but with a brief description in the 
supporting information of Gatti et al., 2015). The DIAMOND code (Putz \& Brandenburg, 1997-2012) was employed to draw all the ball-and-stick pictures.

5. SF view of the spin delocalization and polarization mechanisms in the end-on azido copper(II) dinuclear complex

\subsection{Spin density populations}

QTAIM theoretical net charges $q(\Omega)$ and spin populations $\operatorname{SP}(\Omega)$ for selected atoms or group of atoms for this complex are listed in Table 1. Hereinafter, in the text, tables and figures the term $\mathrm{Cu}^{I I}$ cation(s) is replaced by $\mathrm{Cu}{ }^{I I}$ atom(s) whenever properties of the QTAIM basin(s) or of those derived from Mulliken's partitioning are referred to. In Table 1, QTAIM results are compared with those obtained by Aebersold et al. (1998) from PND data refinement and DFT calculations. Since these latter used a standard Mulliken's population-analysis approach, Mulliken's atomic spin populations, $\mathrm{SP}_{\mathrm{M}}(\Omega)$, are also reported from our calculations, for the sake of comparison. The theoretical data shown in Table 1 demonstrate a large dependency on the method (CASSCF or DFT), DFT functional and basis set adopted. The total spin population of $2 e$ is predicted to be $90 \%$ resident on the $\mathrm{Cu}$ atoms by the $\operatorname{CASSCF}(6,6)$ approach, while this value is reduced to 63 and $43 \%$ by our and the Aebersold et al. (1998) DFT Mulliken estimates, respectively. Aebersold et al. (1998) use a TZVPtype basis set, different from ours, but of similar quality, while for spin density calculations they adopt a local rather than a hybrid DFT functional. The discrepancy between the two DFT results should thus be ascribed mainly to the quite different kind of DFT functional and, possibly, also to the use of a simplified model structure (tert-butyl groups replaced by $\mathrm{H}$ atoms) in the Aebersold et al. (1998) calculations. The PND estimate, $78 \%$ of spin population resident on the $\mathrm{Cu}$ atoms, is intermediate between the CASSCF $(6,6)$ and our DFT result, but closer to the former. Note that the much higher net positive charge of the $\mathrm{Cu}$ atom predicted by $\operatorname{CASSCF}(6,6)(q=$ $1.433 e)$, relative to our DFT estimate $(q=1.092 e)$, may affect the spin population also. QTAIM and Mulliken's spin populations are close to each other, when using the same wavefunction method (compare the data in columns 3 and 4 of Table 1). Yet, their small differences are capable of changing the sign of the population from positive to negative or vice versa, when the populations are small in magnitude (e.g. for the central atom of the azido ligand, i.e. $\mathrm{N} 2$ ).

Overall, the CASSCF $(6,6)$ method predicts that $c a 5.1 \%$ of the spin population is delocalized over the azido ligands and the remaining $4.4 \%$ over the pyridine $\mathrm{N}$ atoms, while other atoms in the system bear almost negligible spin populations. Spin delocalization is about four times as large at the UB3LYP level on both the azido and the pyridine $\mathrm{N}$ atoms, while it is predicted to be about 2.5 times as large by PND. Exchangecorrelation functionals, including the hybrid ones, like UB3LYP, are indeed known to be unable to reproduce the CASSCF spin densities accurately (Boguslawski et al., 2011).
Instead, CASSCF wavefunctions, with an adequate active space, are definitely much closer to the spin densities obtained through density-matrix renormalization group (DMRG) methods, which may be considered as an (almost) exact reference for such densities (Boguslawski et al., 2012). Besides, SD distributions are known to also depend on the approximate exchange-correlation density functional if transition metal complexes containing non-innocent ligands are considered (Conradie \& Ghosh, 2007; Boguslawski et al., 2011). In our work on both the EO and the EE complexes, the UB3LYP method was selected for the sake of comparison with previous studies on the EE complex (Aronica et al., 2007; Deutsch et al., 2014) and for the need to use the same DFT functional to properly compare the spin densities in the EO and EE complexes.

Assuming, therefore, $\operatorname{CASSCF}(6,6)$ as the best theoretical estimated SD among those discussed here for the EO complex, it is concluded that both UB3LYP and the local functional adopted by Aebersold et al. (1998) exaggerate the extent of spin delocalization in the complex. Yet these methods are able to predict almost correctly the relative weight of spin delocalization among the ligands of the system. In particular, there is a general consensus for $\mathrm{N} 2$ having the smallest spin population in the azido group. The smaller positive charge of $\mathrm{Cu}$ in the UB3LYP calculation may also be related to the excessive spin delocalization to ligands, which causes, as a reaction mechanism, the formation of small negative SD regions between the $\mathrm{Cu}$ and its bonded $\mathrm{N}$ atoms. Such spin polarization mechanisms should favour partially covalent rather than pure dative $\mathrm{Cu}-\mathrm{N}$ bonding, leading to a decreased positive charge on $\mathrm{Cu}$, relative to $\operatorname{CASSCF}(6,6)$.

The CASSCF $(6,6)$ estimates are closer to the experimental PND results, but still far from them. Shortcomings of the PND data refinement might explain the observed discrepancies, in particular, the higher spin delocalization found by PND. Indeed, only a very small data set of 152 magnetic structure factors could be considered by Aebersold et al. (1998) in the refinement and an MM including either only one spherical or only one angular Slater atomic function on each atom could be adopted. Convergence of CASSCF estimates with increasing computational quality should, however, also be examined. Increasing the size of the active space from $\operatorname{CASSCF}(6,6)$ to CASSCF $(6,8)$ and CASSCF $(6,10)$ models does not influence significantly either the energy or the atomic SD populations, despite the number of included configurations being increased from 225 to 1960 and 9450, respectively. For instance, the energy is lowered by 0.00012 [CASSCF $(6,8)]$ and 0.00017 a.u. [CASSCF $(6,10)]$, while the SD populations of the $\mathrm{Cu}$ atom, of the azido group and of the pyridine $\mathrm{N}$ atoms all remain unaltered within $0.0005 e$ in both model calculations. At the CASSCF $(6,10)$ level, small changes are found for the individual SD populations in the azido group, the largest changes being $-0.008 e$ for $\mathrm{N} 3$ and $0.006 e$ for the $\mathrm{N} 2$ atoms. On the other hand, increasing the number of correlated electrons is instead a far more delicate issue. To ensure a selection compatible with the symmetry of the system, this number needs to be raised from 6 to at least 14 (two $\pi$ electrons for 
each pyridine unit), leading to too demanding, if not impossible, computations if one wishes to retain a basis set size of sufficient quality for treating electron correlation adequately. CASSCF models with an intermediate number of active electrons ( 8 and 10) have nonetheless been considered. The $\operatorname{CASSCF}(10,10)$ model leads to small asymmetries in the SD populations, with an enhanced SD population for the pyridine $\mathrm{N}$ (only for those two pyridine units having their electrons correlated) and a slow decrease of the $\mathrm{Cu}$ SD population $(0.85 e)$. Despite being physically incorrect, these CASSCF calculations with an 'intermediate' number of active electrons suggest that increasing their number to $14 e$ could lead to an even closer agreement between CASSCF and PND atomic SD populations.

Weighing up the rapid convergence of the CASSCF tests with a fixed number of electrons and increasing orbital active space, and, conversely, the restraints on the number of active electrons, reasonably affordable in CASSCF computations, we have decided to limit our analysis of CASSCF results only to those obtained with the $\operatorname{CASSCF}(6,6)$ model.

\subsection{Spin, magnetic and relaxation density maps and their} atomic Source Function origin

Spin density maps for the $\operatorname{CASSCF}(6,6)$ and UB3LYP calculations, in the least-squares plane of the four ligand $\mathrm{N}$ atoms around each $\mathrm{Cu}^{\mathrm{II}}$, are shown in Fig. 3, along with their magnetic and relaxation densities components.

Comparison of the corresponding maps between the two theoretical methods highlights the much greater spin delocalization on the atoms of the ligands occurring at the UB3LYP relative to the $\operatorname{CASSCF}(6,6)$ level and shows that both the magnetic and relaxation components concur to such an enhancement. Both methods agree that there exists an important spin polarization mechanism in the azido group, with a significantly large region of negative SD around the $\mathrm{N} 2$ atom, reflected in its almost negligible spin population. The negative region around $\mathrm{N} 2$ is obviously due to the SD relaxation component (compare the right and left panels in Fig. 3). However, UB3LYP and CASSCF $(6,6)$ predict quite different portraits for it. The $s_{\text {relax }}$ negative region around $\mathrm{Cu}^{\text {II }}$ atoms seems an artefact of the UB3LYP method and it is in turn responsible for the SD negative region close to the boundaries of the $\mathrm{Cu}$ basins evident in the UB3LYP map (Fig. 3, lower row, left panel). Such a region is totally absent in the $\operatorname{CASSCF}(6,6) \mathrm{SD}$ map (Fig. 3, upper row, left panel), except for a tiny area between $\mathrm{Cu}^{\mathrm{II}}$

Figure 3 and the bridging N1 atom. The UB3LYP and CASSCF $(6,6)$ relaxation SDs also differ with regard to the extent of spin polarization around N2 and the presence in the UB3LYP map of an extended positive region around $\mathrm{N} 1$, which is totally lacking in the $\operatorname{CASSCF}(6,6)$ map. Besides that, the CASSCF $(6,6)$ relaxation SD around the $\mathrm{Cu}^{\mathrm{II}}$ cations displays the same $d_{x^{2}-y^{2}}$ angular shape as the SD (refer to Fig. 2 for axes orientation), whereas a much more rounded, almost spherical, shape is visible in the UB3LYP relaxation SD map.

Table 1 also lists the value of the atomic SD Laplacian (ASDL), $\int_{\Omega} \nabla^{2} s$ dr, while Fig. 4 shows the $\nabla^{2} s$ scalar field isolines in the same plane of Fig. 3. At variance with the atomic electron-density Laplacian, $\int_{\Omega} \nabla^{2} \rho$ dr, ASDL usually differs from zero because the atomic boundary surface is defined in terms of the local zero flux of $\nabla \rho$ and not of that of $\nabla s$. ASDL values provide a measure of whether, on average, a QTAIM atomic basin has a prevailing $\alpha(\mathrm{ASDL}<0)$ or $\beta$ $($ ASDL $>0)$ effect, though its specific effect on a given $r p$ will depend on how the Green's term locally weights the integrand $\nabla^{2} s$ in the $\mathrm{SF}_{\mathrm{S}}$ expression. The $\mathrm{Cu}$ atom, which holds most of the unpaired density, has a negative and the largest (in magnitude) ASDL, indicating a prevailing $\alpha$ effect, while all $\mathrm{N}$ atoms of the ligands, except the terminal $\mathrm{N}$ atoms of the azido groups, have a positive ASDL and a prevailing $\beta$ effect. The features of $\nabla^{2} s$ maps (Fig. 4) provide further details. The capability of the $\mathrm{Cu}$ atom to produce an $\alpha$ effect is due to the dominance of the dotted blue regions $\left(\nabla^{2} s<0\right)$, which are very localized, directed towards the ligands and characterized by high $\left|\nabla^{2} s\right|$ values. The negative $\nabla^{2} s$ region around the copper has the shape of a squeezed $\mathrm{Cu}^{\mathrm{II}} d_{x^{2}-y^{2}}$ atomic orbital surrounded by a large positive $\nabla^{2} s$ region where the copper yields a counteracting $\beta$ effect. However, on average, the $\alpha$ effect prevails, as indicated by the $\mathrm{Cu}$ negative ASDL value.

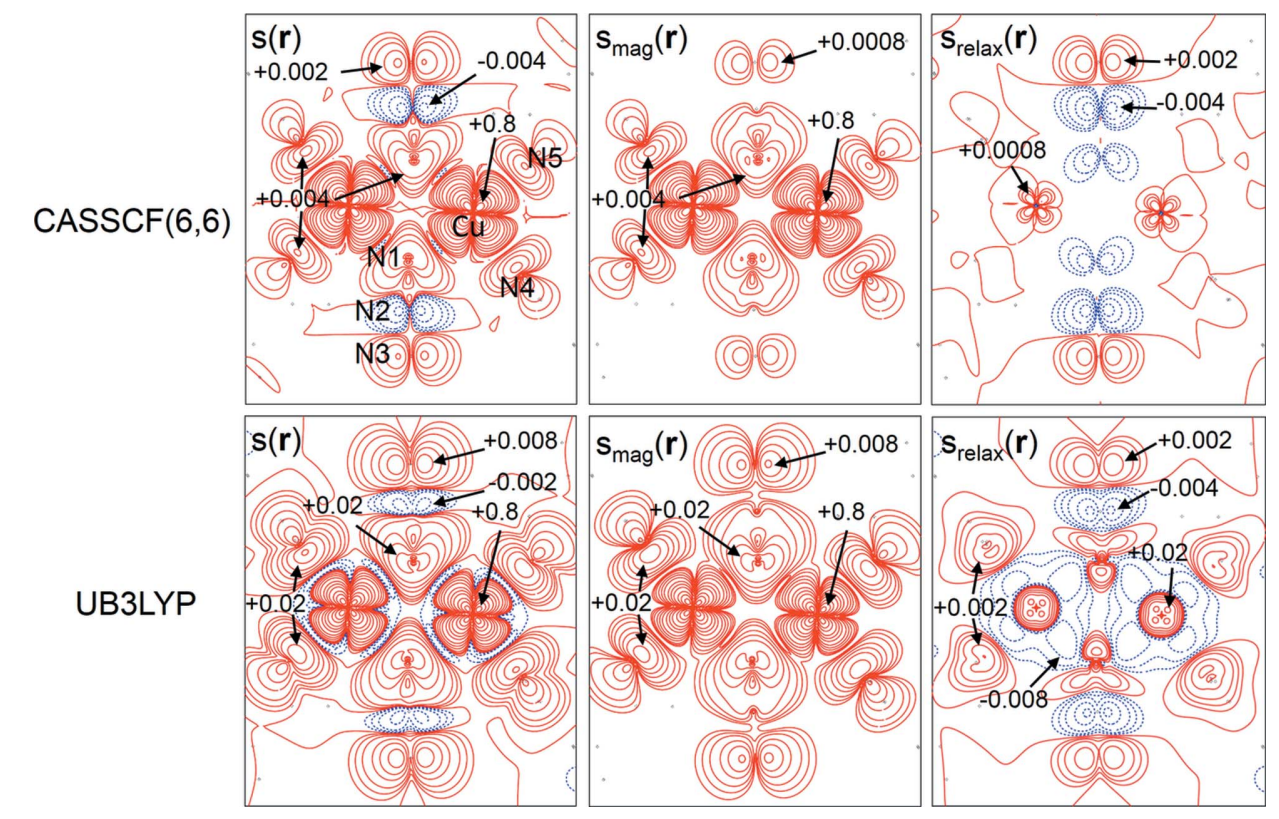

End-on FM complex: CASSCF(6,6) and UB3LYP contour plots of the spin density (left panel) and of its magnetic (central panel) and relaxation (right panel) components in the least-squares plane of the four ligand $\mathrm{N}$ atoms around each $\mathrm{Cu}^{\mathrm{II}}$. The same colour codes and isovalue contours apply as in Fig. 1 . 


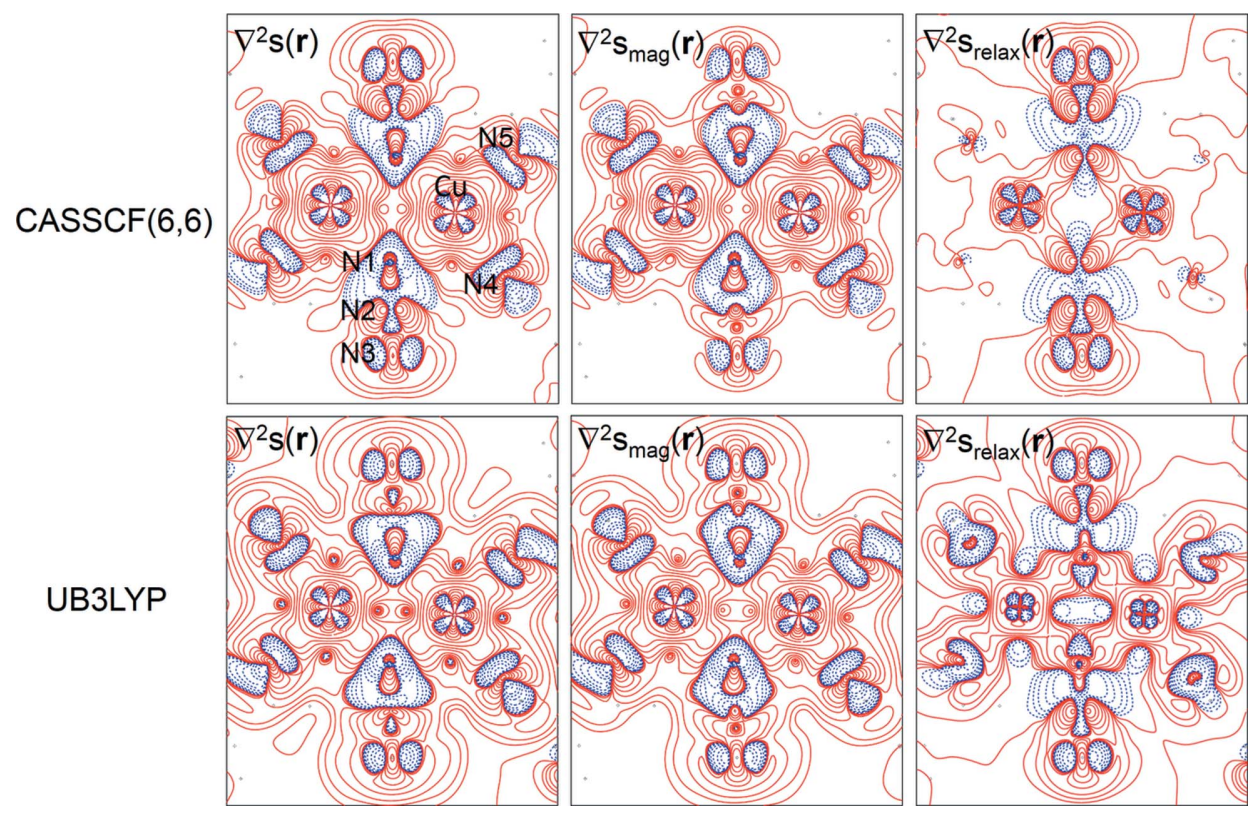

Figure 4

End-on FM complex: CASSCF(6,6) and UB3LYP contour plots of the spin density Laplacian (left panel) and of its magnetic (central panel) and relaxation (right panel) components in the least-squares plane of the four ligand $\mathrm{N}$ atoms around each $\mathrm{Cu}^{\mathrm{II}}$. The same colour codes and isovalue contours apply as in Fig. 1. average, over the $\beta$ effect regions close to their nuclei. This holds particularly true at the CASSCF $(6,6)$ level. The $\alpha$ effect regions of the $\mathrm{N}$ atom linked to $\mathrm{Cu}^{\mathrm{II}}$ are those able to determine delocalization of the unpaired electron density. The central atom of the azido ligand has also a prevailing $\beta$ effect, whereas the terminal $\mathrm{N}$ atom behaves in an opposite manner as a result of its $\pi$-like and dominating $\alpha$ effect regions. Spin polarization in the azido ligands is also evident from the opposing average spin density source effects produced by the terminal and the other two $\mathrm{N}$ atoms of the group, with these latter prevailing when the whole azido group is considered (Table 1, ASDL $>0$ for the azido group). UB3LYP and $\operatorname{CASSCF}(6,6)$ spin density Laplacian maps differ for their relaxation components, which

Fig. 4 also shows that the bridging and pyridine $\mathrm{N}$ atoms have negative $\nabla^{2} s, \alpha$ effect regions, pointing towards copper and wider than those of $\mathrm{Cu}$ atoms, but unable to prevail, on are significantly different in shape and local scalar magnitude, while the magnetic components are, at least as for their shape, closer to each other.
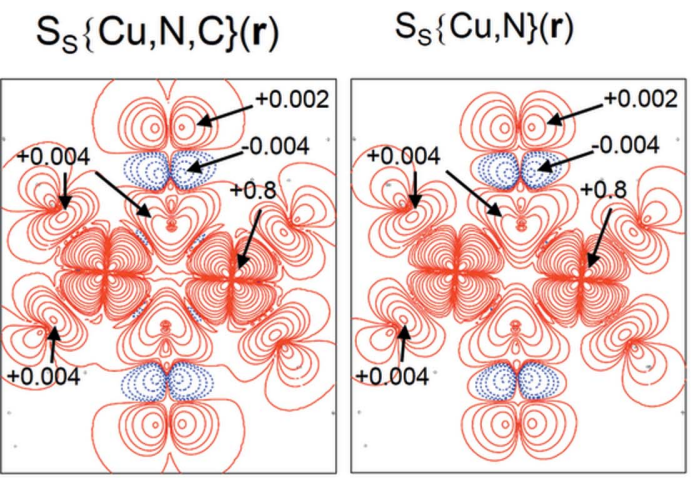

$\mathrm{S}_{\mathrm{s}}\{\mathrm{Cu}\}(\mathbf{r})$

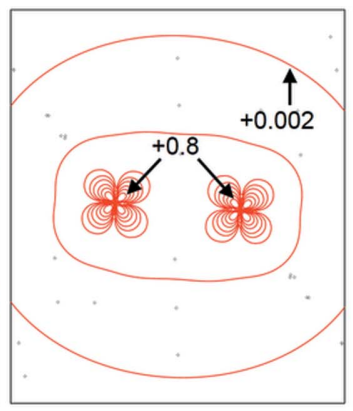

$\operatorname{CASSCF}(6,6)$
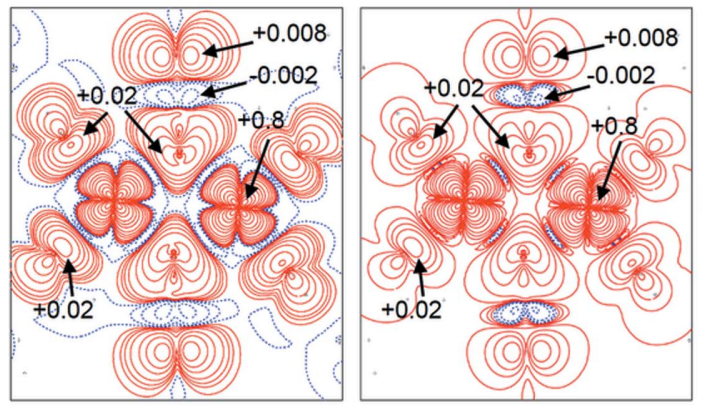

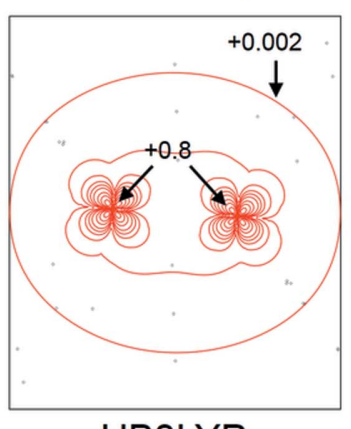

UB3LYP

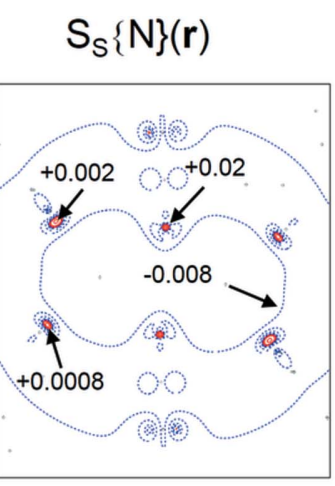

$\mathrm{S}_{\mathrm{S}}\left\{\mathrm{N}_{\mathrm{azide}}\right\}(\mathbf{r})$
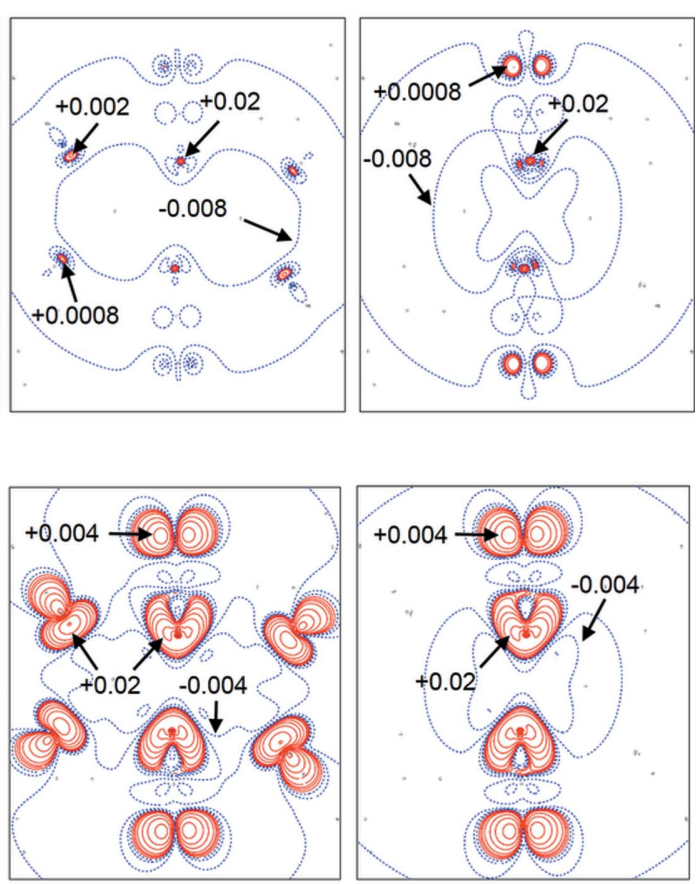

Figure 5

End-on FM complex: CASSCF(6,6) (up) and UB3LYP (down) contour plots of the SF reconstructed partial spin densities, $S_{\mathrm{s}}\left(\Omega_{\text {subset }}\right)(\mathbf{r})$ in the leastsquares plane of the four ligand $\mathrm{N}$ atoms around each $\mathrm{Cu}^{\mathrm{II}}$. The corresponding magnetic and relaxation components are shown in Figs. S1-S2. In the maps, $\{\mathrm{Cu}, \mathrm{N}, \mathrm{C}\}$ denotes the subset of the atoms of the system including the two $\mathrm{Cu}$ atoms, all $\mathrm{N}$ atoms and the $\mathrm{C}$ atoms of the pyridine rings. Similar labelling is used for the group of atoms included in the other listed subsets, with $\mathrm{N}_{\text {azido }}$ denoting an $\mathrm{N}$ atom of the azido groups only. The same colour codes and isovalue contours apply as in Fig. 1. 
SF reconstructed partial densities (Fig. 5, Figs. S1-S2 of the supporting information) provide a cause-effect view of the SD and of its components. Group subsets $\{\mathrm{Cu}, \mathrm{N}, \mathrm{C}\},\{\mathrm{Cu}, \mathrm{N}\},\{\mathrm{N}\}$, $\left\{\mathrm{N}_{\text {azido }}\right\}$ are considered, where $\{\mathrm{Cu}\},\{\mathrm{N}\}$ and $\{\mathrm{C}\}$ denote the subsets including the two $\mathrm{Cu}$ atoms, all $\mathrm{N}$ atoms and all $\mathrm{C}$ pyridine rings atoms, respectively, while $\left\{\mathrm{N}_{\text {azido }}\right\}$ denotes only the $\mathrm{N}$ atoms of the azido ligands. Figs. S1-S2 compare these reconstructed partial spin densities with their corresponding magnetic and relaxation density components, while Fig. 6 reports such a comparison also for the subsets including the bridging, $\left\{\mathrm{N} 1, \mathrm{~N}^{\prime}\right\}$, the central, $\left\{\mathrm{N} 2, \mathrm{~N} 2^{\prime}\right\}$, and the terminal, $\left\{\mathrm{N} 3, \mathrm{~N}^{\prime}\right\}$, atoms of the azido groups.

Including all atoms in the atomic subset, except the pyridine $\mathrm{H}$ and those of the tert-butyl groups, leads to $\mathrm{SF}\{\mathrm{Cu}, \mathrm{N}, \mathrm{C}\}$ reconstructed partial density maps almost indistinguishable from their primitive maps, indicating that the contributions from the excluded atoms are negligible ( $c f$. Figs. and 5). The only noticeable differences concern the peripheral regions of the map and this consideration holds true both for the SD and for its magnetic and relaxation components (Fig. S1). When the pyridine $\mathrm{C}$ atoms are also excluded from the reconstruction, important differences begin to emerge, essentially at the UB3LYP level. For instance, the negative SD region surrounding the $\mathrm{Cu}$ atoms and close to their atomic boundaries appears to be mostly due to the effect of the pyridine $\mathrm{C}$ atoms (compare the first and second lower panels in Fig. 5 and the second row versus the fourth row panels in Fig. S1). The negative density regions in the relaxation SD map contract and so do the regions of very high positive density around the $\mathrm{Cu}^{\mathrm{II}}$ cations in the magnetic density map (Fig. S1, second and fourth row panels). The fact that only the UB3LYP densities appear to be significantly affected by the exclusion of the SF contributions of relatively remote atoms in the molecular regions mapped in Figs. 5 and S1 further confirms that the UB3LYP likely exaggerates spin delocalization mechanisms. Interestingly, this appears true not only in the direction from the paramagnetic atoms to the ligands, but also the other way round. This reversed direction may be only appreciated through the SF analysis and within a cause-effect context. The third panel in the first (CASSCF) and second (UB3LYP) rows of Fig. 5 illustrates the SD source from the $\mathrm{Cu}$ atoms. As expected, it is the most relevant source and the one that imparts the $d_{x^{2}-y^{2}}$ symmetry to the total SD. It is always positive (in this plane) and it is dominated by the magnetic component (see Fig. S2). The $\mathrm{Cu}+\mathrm{Cu}^{\prime} \mathrm{SD}$ source is larger in magnitude at the CASSCF level (compare the UB3LYP and CASSCF contour level values in Fig. 5) since, differently from CASSCF, the UB3LYP model predicts, in the relaxation SD map, large regions of opposing negative SD sources besides those of positive sign close to the $\mathrm{Cu}$ nuclei. The fourth and
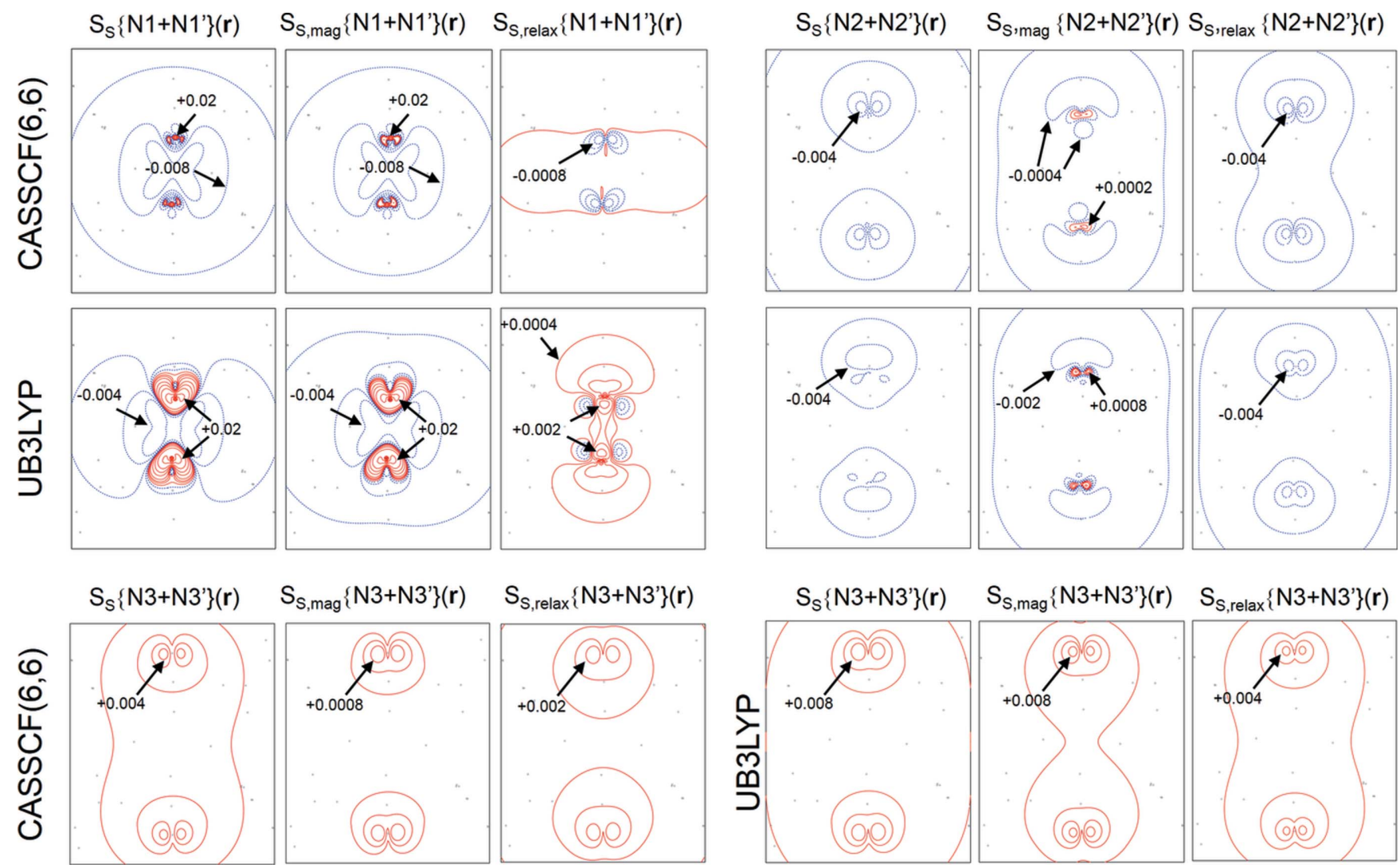

Figure 6

End-on FM complex: CASSCF $(6,6)$ and UB3LYP contour plots of the partially SF reconstructed spin densities, $S_{\mathrm{s}}\left(\Omega_{\text {subset }}\right)(\mathbf{r})$, and of its magnetic, $S_{\mathrm{s}, \text { mag }}$ $\left(\Omega_{\text {subset }}\right)(\mathbf{r})$, and relaxation, $S_{\mathrm{s}, \text { relax }}\left(\Omega_{\text {subset }}\right)(\mathbf{r})$, components in the least-squares plane of the four ligand $\mathrm{N}$ atoms around each Cu ${ }^{\mathrm{II}}$. In the maps, $\{\mathrm{N} 1+$ $\left.\mathrm{N} 1^{\prime}\right\},\left\{\mathrm{N} 2+\mathrm{N}^{\prime}\right\}$ and $\left\{\mathrm{N} 3+\mathrm{N}^{\prime}\right\}$ denote the subsets of the atoms of the system, including the bridging, the central and the terminal $\mathrm{N}$ atoms of the azido groups, respectively. The same colour codes and isovalue contours apply as in Fig. 1. 
fifth panels in the first (CASSCF) and second (UB3LYP) rows of Fig. 5 reveal, respectively, the SF role played by all the $\mathrm{N}$ atoms of the system and that played by the $\mathrm{N}$ atoms of the azido ligands only. At the CASSCF $(6,6)$ level, the $\mathrm{N}$ atoms act as diffuse negative sources, except for small regions of, higher in magnitude, positive SD around their nuclei. The diffuse negative sources are small but able to counteract the positive source from the $\mathrm{Cu}$ atoms in the region around $\mathrm{N} 2$ and in a tiny region between $\mathrm{Cu}$ and $\mathrm{N} 1$. There is also a noticeable counter effect of the pyridine $\mathrm{N}$ atoms on the positive sources around the $\mathrm{N}$ nuclei of the azido ligands. When only the $\left\{\mathrm{N}_{\text {azido }}\right\}$ sources are considered, the positive SD regions around their nuclei clearly increase in size. Conversely, at the UB3LYP level, the $\mathrm{N}$ atoms yield larger and definitely more delocalized regions of positive SD sources around their nuclei, with the positive sources around those of the azido ligands being scarcely affected by the presence or not of the sources from the pyridine $\mathrm{N}$ atoms. The two sets of $\mathrm{N}$ atoms appear to be more independent, relative to the CASSCF model, suggesting that higher delocalization of the unpaired electrons does not necessarily imply a greater connection between them. The positive $\mathrm{SD}$ sources around the $\mathrm{Cu}^{\mathrm{II}}$-bonded $\mathrm{N}$ are directed toward the $\mathrm{Cu}^{\mathrm{II}}$ cation(s) they are linked to, thereby imparting a special split-lobe shape to the source of the bridging atoms of the azido ligands. The UB3LYP and CASSCF descriptions of the sources from the $\{\mathrm{N}\}$ and the $\left\{\mathrm{N}_{\text {azido }}\right\}$ subsets largely differ both for the SD source (Fig. 5) and for that of its magnetization and relaxation components (Fig. S2). The magnetic component imparts the shape of the SD sources at the UB3LYP level, while at the CASSCF level, the relaxation SD plays a more decisive role, especially for the central and terminal atoms of the azido groups.
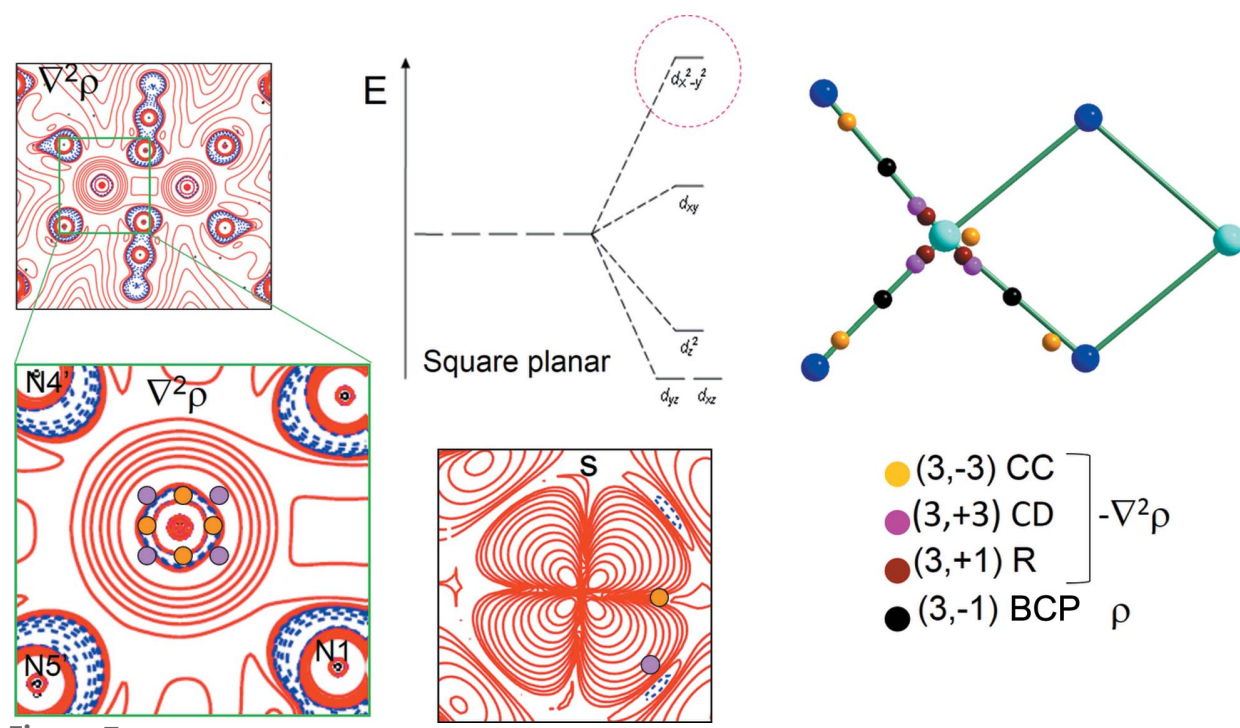

Figure 7

$\mathrm{Cu}$ atom $3 d$ electron asphericity and its electron-density Laplacian and electron spin density image in the end-on FM complex. Orbital ordering for a metal atom in a square-planar arrangement of ligands (top middle). The Laplacian of the CASSCF $(6,6)$ electron density (top left) in the plane of the $\mathrm{N}$ atoms, with an enlargement (bottom left) of the VSCC and VSCD of $\mathrm{Cu}^{\mathrm{II}}$. CASSCF $(6,6)$ spin density (bottom middle). The locations of the unique CPs of $-\nabla^{2} \rho$ and $\rho$, whose properties are reported in Table 2, are shown on the right of the figure. Locations of the charge concentrations (CC) and depletions (CD) are shown in the electron-density Laplacian and in the electron spin density maps around $\mathrm{Cu}^{\mathrm{II}}$.
Fig. 6 enables one to distinguish neatly the different roles played by the bridging, the central and the terminal $\mathrm{N}$ atoms of the azido groups. The bridging atoms yield both local positive sources and diffuse negative sources, with a spin polarization mechanism of sources with its bonded atoms. The central atoms, instead, yield only diffuse negative sources and the terminal atoms diffuse and small positive sources. The source from the bridging $\mathrm{N}$ atoms is dominated by the magnetic component in both the $\operatorname{CASSCF}(6,6)$ and the UB3LYP calculations, but largely differs in shape and magnitude for the two models. The positive source around the $\mathrm{N}$ atom, directed towards the two linked metal atoms, is much larger in size at the UB3LYP level. On the contrary, the source from the central atoms is always dominated by the relaxation component, leading to an evident negative source for the SD. oth this component source and the SD source have a $\pi$-like shape. This is reminiscent of the symmetry of the unoccupied ecular orbitals (MOs) resulting from the mixing of the the $d_{x^{2}-y^{2}}$ metal orbitals in a fragment orbital approach interpretation of the SD in the EO the fexersold et al., 1998). These MOs, unoccupied at role in the spin polarization ligand, when the system as a whole is considered (Aebersold $e t$ al., 1998). Finally, for the terminal atoms, both density components are relevant and co-operate to make this atom act as a small positive source at both computational levels. Yet, CASSCF $(6,6)$ predicts the relaxation component as more relevant, while it is the magnetic component that prevails at the UB3LYP level. To conclude, we observe that, except for a large part of the $\mathrm{Cu}$-atom basins, the local SD is in general quite different from that due to the source of the closest atom or group of atoms, a difference which provides a local measure of the co-operating effects inherent to the electron spin delocalization and polarization mechanisms. In other words, except for $\mathrm{Cu}$ atoms, the sources of the other atoms in the systems are unable to explain, often even qualitatively, the SD distribution around them. The effect of other atoms is, in general, far from being negligible.

\section{3. $\mathrm{Cu}^{\prime \prime} 3 d$ electron asphericity and its electron-density Laplacian and electron spin density image}

In the EO complex, the $\mathrm{Cu}^{\text {II }}$ cation is fourfold coordinated in an almost square-planar arrangement of ligands. In this geometry and for a $\mathrm{Cu}^{\mathrm{II}}$ cation ( $d^{9}$ configuration), the $d_{x^{2}-y^{2}}$ orbital is the magnetic singly occupied orbital, according to crystal field theory (Fig. 7). The 
Table 2

End-on (EO) FM complex: spin, $s(\mathbf{r})$, magnetic, $s_{\text {mag }}(\mathbf{r})$, relaxation, $s_{\text {relax }}(\mathbf{r})$, density and Laplacian of the electron density, $\nabla^{2} \rho(\mathbf{r})$, properties at bond critical points (BCPs) and at some relevant $-\nabla^{2} \rho$ critical points (CPs) along the $\mathrm{Cu}-\mathrm{Cu}, \mathrm{Cu}-\mathrm{N} 1, \mathrm{Cu}-\mathrm{N} 4$ and $\mathrm{Cu}-\mathrm{N} 5$ bonds.

CASSCF $(6,6)$ data (UB3LYP data in parentheses); if not otherwise stated, all values are in atomic units.

\begin{tabular}{|c|c|c|c|c|c|c|}
\hline Bond $\uparrow$ & $\mathrm{CP}$ & $R_{\mathrm{Cu}}(\AA)$ & $\nabla^{2} \rho(\mathbf{r})$ & $s(\mathbf{r})$ & $s_{\text {mag }}(\mathbf{r})$ & $s_{\text {relax }}(\mathbf{r})$ \\
\hline $\mathrm{Cu}-\mathrm{Cu}^{\prime}$ & $-\nabla^{2} \rho(3,-3), \mathrm{CC}$ & $0.277(0.278)$ & $-104.95(-94.76)$ & $0.000(0.011)$ & $0.000(0.000)$ & $0.000(0.011)$ \\
\hline \multirow[t]{3}{*}{$\mathrm{Cu}-\mathrm{N} 1$} & $-\nabla^{2} \rho(3,+3), \mathrm{CD}$ & $0.429(0.434)$ & $19.33(18.04)$ & $0.413(0.278)$ & $0.412(0.274)$ & $0.001(0.004)$ \\
\hline & $-\nabla^{2} \rho(3,+1), \mathrm{R}$ & $0.292(0.288)$ & $-28.37(-41.13)$ & $1.030(0.750)$ & $1.029(0.733)$ & $0.001(0.017)$ \\
\hline & $\rho, \mathrm{BCP}$ & $0.939(0.967)$ & $0.36(0.28)$ & $0.007(0.002)$ & $0.007(0.003)$ & $0.000(-0.001)$ \\
\hline \multirow{3}{*}{$\mathrm{Cu}-\mathrm{N} 4$} & $-\nabla^{2} \rho(3,+3), \mathrm{CD}$ & $0.430(0.434)$ & $19.41(18.13)$ & $0.416(0.286)$ & $0.416(0.282)$ & $0.000(0.004)$ \\
\hline & $-\nabla^{2} \rho(3,+1), \mathrm{R}$ & $0.292(0.288)$ & $-29.27(-42.04)$ & $1.051(0.792)$ & $1.050(0.774)$ & $0.001(0.018)$ \\
\hline & $-\nabla^{2} \rho(3,-3), \mathrm{CC}$ & $1.597(1.595)$ & $-2.74(-2.38)$ & $0.008(0.036)$ & $0.008(0.032)$ & $0.000(0.004)$ \\
\hline & $-\nabla^{2} \rho(3,+1), \mathrm{R}$ & $0.292(0.288)$ & $-28.87(-41.96)$ & $1.057(0.788)$ & $1.056(0.770)$ & $0.001(0.018)$ \\
\hline & $-\nabla^{2} \rho(3,-3), \mathrm{CC}$ & $1.593(1.591)$ & $-2.69(-2.35)$ & 0.008 & $0.008(0.030)$ & $0.000(0.003)$ \\
\hline & $\rho, \mathrm{BCP}$ & $0.923(0.953)$ & $0.39(0.29)$ & $0.008(0.001)$ & $0.008(0.002)$ & $0.000(-0.001)$ \\
\hline
\end{tabular}

$\dagger-\nabla^{2} \rho$ CP legend: $(3,-3)$ charge concentration, CC; $(3,+3)$ charge depletion, $\mathrm{CD} ;(3,+1)$ ring, $\mathrm{R}$.

topology of the Laplacian of the electron density may validate whether such a simplified model theory is also reflected in the properties of an observable (Gatti, 2005). In particular, the asphericity of the $3 d$ electron distribution around a metal atom, due to an unequal occupation of its $3 d$ valence orbitals, may be neatly revealed this way (Bianchi et al., 1996; Macchi \& Sironi, 2003; Gatti, 2005; Lo Presti \& Destro, 2008). Topological analysis of $-\nabla^{2} \rho$ in the valence-shell charge concentration (VSCC; Bader, 1990) and valence-shell charge depletion (VSCD; Bader, 1990) regions of the $\mathrm{Cu}$ atoms finds four $(3,+3)$ $-\nabla^{2} \rho$ minima, i.e. charge depletions (CDs), lying almost on the $\mathrm{Cu}-\mathrm{N}_{\text {py }}$ and on the $\mathrm{Cu}-\mathrm{N}_{\text {bridging internuclear axes and }}$ four $(3,-3)-\nabla^{2} \rho$ maxima, i.e. charge concentrations (CCs), lying in between the CDs (Fig. 7).

Thus, the CDs, located at $0.27 \AA$ from the $\mathrm{Cu}$ nucleus, may be associated with the lobes of the formally singly occupied $d_{x^{2}-y^{2}}$ orbital and the CCs, located at $0.43 \AA$ from the $\mathrm{Cu}$ nucleus, with the lobes of the formally doubly occupied $d_{x y}$ orbital. If such an association is plausible, one would anticipate a large SD value at the CDs and much smaller values at the CCs. Conversely, CDs should be characterized by positive and low $\nabla^{2} \rho$ values compared with the high $\left|-\nabla^{2} \rho\right|$ magnitudes at CCs, illustrating that the electron-density Laplacian and the electron SD distributions provide complementary pictures. Where one scalar field is concentrated, the other is depleted and vice versa, since a higher SD value implies a region dominated by a metal orbital with low electron occupation, while a higher $\mathrm{CC}$, hence a higher $\left|-\nabla^{2} \rho\right|$ magnitude, implies a region dominated by a metal orbital with higher or maximal orbital occupation.

The results shown in Table 2 neatly confirm our hypothesis. For instance, at the $\operatorname{CASSCF}(6,6)$ level, the $s$ and $\nabla^{2} \rho$ values are 0.413 and 19.33 a.u. at the $\mathrm{CD}$ along the $\mathrm{Cu}-\mathrm{N} 1$ axis, relative to the 0.001 and -104.95 a.u. values at the $\mathrm{CC}$ lying along the $\mathrm{Cu}-\mathrm{Cu}^{\prime}$ axis. Qualitatively similar results are found at the UB3LYP level (Table 2), but the $s$ values (0.278 and 0.011 a.u.) are decreased at the $\mathrm{CD}$ and significantly increased at the $\mathrm{CC}$, with respect to the electron-correlated wavefunc- tion. High SD at the CDs is almost all due to the magnetic term, while the very low SD at the CCs has essentially a relaxation density origin (Table 2). Since the UB3LYP model exaggerates both the spin delocalization to the ligands and the local spin relaxation mechanisms, SD turns out to be smaller at $\mathrm{CDs}$ and larger at $\mathrm{CCs}$ close to the $\mathrm{Cu}$ nucleus, relative to the CASSCF $(6,6)$ values. A $(3,+1)-\nabla^{2} \rho$ ring $(R$, Table 2$)$ critical point is also found along all the $\mathrm{Cu}-\mathrm{N}_{\text {ligand }}$ axes and located at a distance from the $\mathrm{Cu}$ nucleus very close $(0.28 \AA)$ to that of the CCs. At this point, the $\nabla^{2} \rho$ is negative, as at the CC $r p$, but significantly lower in magnitude, while the SD is extremely large [for instance, $\nabla^{2} \rho=-28.37$ a.u. and $s=1.030$ a.u. at the ring point along $\mathrm{Cu}-\mathrm{N} 1$, at the $\operatorname{CASSCF}(6,6)$ level]. This ring $\mathrm{CP}$ region is also associated with the $d_{x^{2}-y^{2}}$ orbital. These additional data further corroborate our analysis relating $\mathrm{Cu}$ metal asphericity with the SD and the Laplacian of electrondensity features around the metal. It is also worth noting that both the SD and the SD Laplacian portraits of the $\mathrm{Cu}^{\mathrm{II}}$ cation provide a rigorous picture of an orbital density, one based on quantum observables and not on a model.

Also reported in Table 2 are the $s$ and $\nabla^{2} \rho$ values at the $\mathrm{Cu}-\mathrm{N}$ BCP locations and at the CCs lying in the VSCCs of the pyridine and bridging $\mathrm{N}$ atoms. These $\mathrm{CCs}$, one for each $\mathrm{N}$ atom except for N1, which has two equivalent CCs of this kind, are all almost aligned in a key-lock arrangement, with the corresponding $\mathrm{CDs}$ located close to the $\mathrm{Cu}$ nucleus. At the CASSCF $(6,6)$ level, the SD at the $\mathrm{N}$-atom CCs and at the $\mathrm{Cu}-\mathrm{N}$ BCP locations is low, about two orders of magnitude lower than at the $\mathrm{Cu} \mathrm{CDs}$ and essentially due to the magnetic component (Table 2). On the other hand, UB3LYP predicts $\mathrm{SD}$ values that are three times higher at the $\mathrm{N}$-atom $\mathrm{CCs}$ and half as high at the $\mathrm{BCPs}$, relative to $\operatorname{CASSCF}(6,6)$. Yet, also for the UB3LYP model, the SD at these critical points is largely dominated by the magnetic component (Table 2). Note that all UB3LYP $\mathrm{Cu}-\mathrm{N}$ BCP locations are displaced towards the $\mathrm{N}$ nuclei, relative to the $\operatorname{CASSCF}(6,6)$ wavefunction. This fact corroborates the link between the UB3LYP excessive spin delocalization to ligands and the decreased dative or increased 

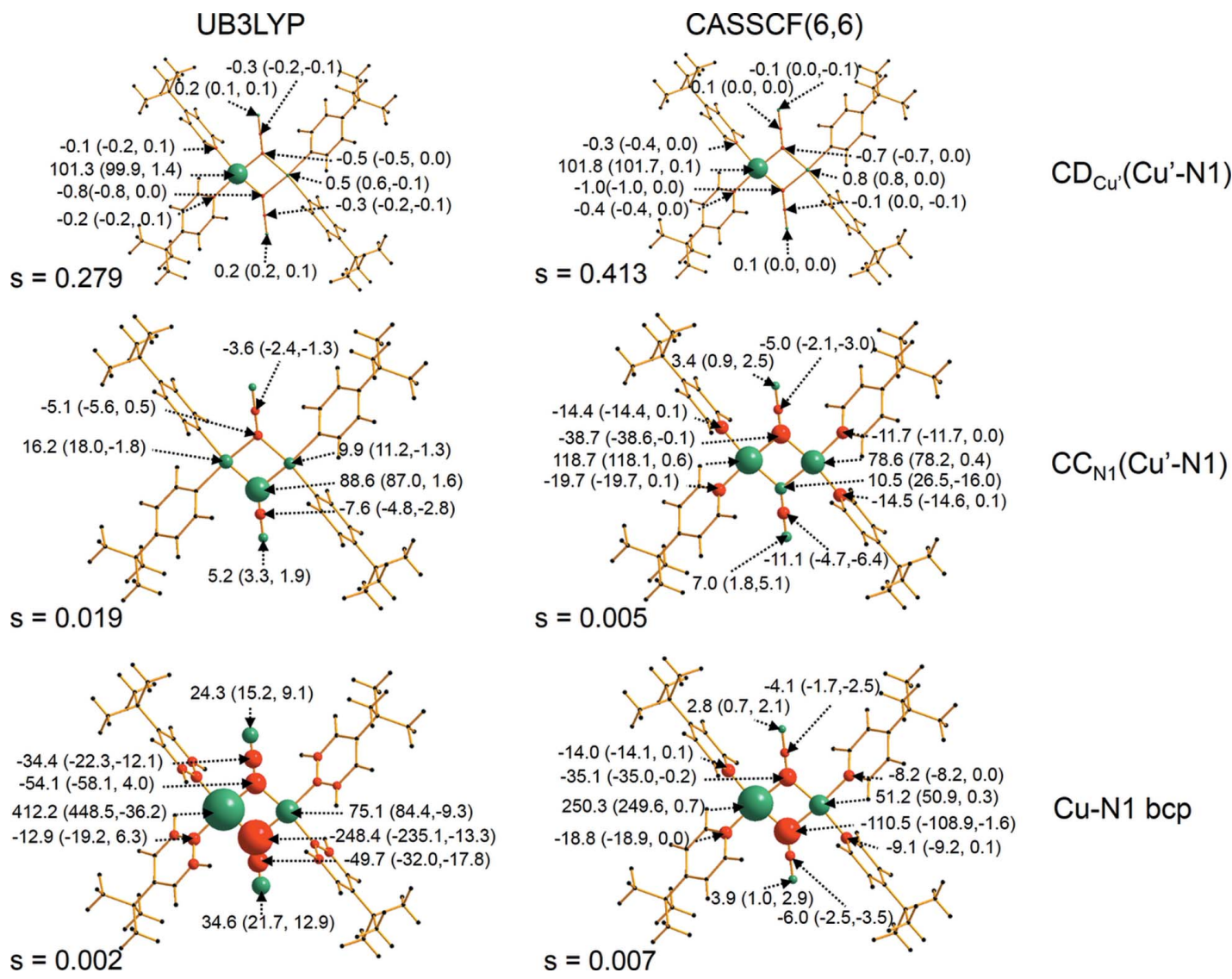

Figure 8

End-on FM complex: UB3LYP and CASSCF(6,6) SF percentage contributions to the electron spin density $s$ (magnetic and relaxation components in parentheses) at selected reference points, rps (Table 2). Rps: $\mathrm{CD}_{\mathrm{Cu}^{\prime}}\left(\mathrm{Cu}^{\prime}-\mathrm{N} 1\right)$ denotes the charge depletion in the valence-shell charge depletion (VSCD) of the $\mathrm{Cu}^{\prime}$ atom and lying along $\mathrm{Cu}^{\prime}-\mathrm{N} 1$, while $\mathrm{CC}_{\mathrm{N} 1}\left(\mathrm{Cu}^{\prime}-\mathrm{N} 1\right)$ denotes the charge concentration in the valence-shell charge concentration (VSCC) of the $\mathrm{N} 1$ atom and lying almost along $\mathrm{Cu}^{\prime}-\mathrm{N} 1$. The reported $s$ values at $r p s$ are in a.u. Green (red) atomic balls denote an $\alpha(\beta)$ effect on the spin density at the reference point. SF percentage contributions are positive or negative whether they concur or oppose to reconstruct the corresponding spin density value at the $r p$.

covalent character of the $\mathrm{Cu}-\mathrm{N}$ bonding, leading to a smaller positive charge on $\mathrm{Cu}$ and to the $\mathrm{BCP}$ displacement towards $\mathrm{N}$ atoms.

\subsection{Source Function analysis at selected reference points}

In §5.2, spin, magnetic and relaxation densities in the EO complex have been shown and the way these densities are determined from their atomic or atomic group sources, in the plane of the $\mathrm{N}$-atom ligands around the $\mathrm{Cu}^{\mathrm{II}}$ atom, has been discussed. In $\S 5.3$, the relationship between $\mathrm{Cu}^{\mathrm{II}} 3 d$ electron asphericity and features of the electron-density Laplacian and electron SD around this atom have been pointed out. Properties at a number of peculiar molecular points have been analysed and some of those points are now considered as convenient $r p$ s for a conventional SF spin density analysis, along with $\mathrm{Cu}^{\prime}, \mathrm{N} 1$ and $\mathrm{N}^{\prime}$ nuclei as additional rps (Figs. 8 and 9). Let us first consider atomic sources at the $\mathrm{CD}$ in the VSCD of $\mathrm{Cu}^{\prime}$ along $\mathrm{Cu}^{\prime}-\mathrm{N} 1 \mathrm{CD}_{\mathrm{Cu}}\left(\mathrm{Cu}^{\prime}-\mathrm{N} 1\right)$ in Fig. 8]. SD is dominated by the source of $\mathrm{Cu}^{\prime}(101.8$ and $101.3 \%$ at the CASSCF and UB3LYP levels, respectively) and essentially by its magnetic component (101.7 and $99.9 \%$ at the CASSCF and UB3LYP levels, respectively). Yet, despite being very small in percentage, sources from the atoms of the azido ligands (in particular atom $\mathrm{N} 1$ ) and from the remote $\mathrm{Cu}$ atom are not negligible in absolute value and being negative, except those from $\mathrm{Cu}$ and from the terminal atoms of the azido ligands, contrast the source from $\mathrm{Cu}^{\prime}$. A $|1 \%|$ value for such contributions amounts to $|0.003|$ a.u. (UB3LYP), which is, for instance, larger than the value (0.002 a.u.) of the total SD at $\mathrm{Cu}^{\prime}-\mathrm{N} 1 \mathrm{BCP}$ and a typical SD value in many regions of the system. These sources, in an analogous manner to the dominant one from $\mathrm{Cu}^{\prime}$, are essentially due to the magnetic component. Their absolute value assesses the local importance of the spin back-delocalization mechanism from the ligands to the paramagnetic centre or, in the case of $\mathrm{Cu}$, the spin delocalization between the paramagnetic centres themselves. Then, being the SD positive at the $r p$, their positive ( $\alpha$ effect) or negative ( $\beta$ effect) sign determines whether they concur or contrast the $\mathrm{SD}$ at this $\mathrm{CD}$. Interestingly, the remote $\mathrm{Cu}$, at variance with the bridging $\mathrm{N}$ atoms, concurs with the $\mathrm{SD}$ at the $\mathrm{Cu}^{\prime} \mathrm{CD}$, in agreement with the two $\mathrm{Cu}^{\mathrm{II}}$ cations being ferromagnetically coupled through the magnetic density.

It is interesting to compare the SF pattern at the $\mathrm{CD}$ in the VSCD of $\mathrm{Cu}^{\prime}$ along $\mathrm{Cu}^{\prime}-\mathrm{N} 1$ with that at the $\mathrm{CC}$ along $\mathrm{Cu}^{\prime}-$ 
$\mathrm{Cu}$ and close to $\mathrm{Cu}^{\prime}$ (not shown in the figure). As discussed earlier, the SD here is almost zero [ -0.00005 a.u., CASSCF $(6,6)]$ or small [0.011 a.u., UB3LYP] and with a nonnull relaxation component only. However, such a negligible or small value is, for both methods, the result of a perfect balance among relatively high magnetic sources that oppose each other to yield an overall null magnetic source. Both $\mathrm{Cu}$ atoms play an $\alpha$ effect, while the bridging and central $\mathrm{N}$ atoms of the azido ligands and the pyridine $\mathrm{N}$ atoms oppose them with a $\beta$ effect almost equal in magnitude. Overall, at an $r p$ related to the magnetic pathway [i.e. at $\left.\mathrm{CD}_{\mathrm{Cu}}\left(\mathrm{Cu}^{\prime}-\mathrm{N} 1\right)\right]$, the magnetic sources dominate and the SD is large, while at one related to a doubly occupied orbital (i.e. the $\mathrm{CC}$ along $\mathrm{Cu}^{\prime}-\mathrm{Cu}$; Table 2) the magnetic sources, despite being relatively high and definitely much more delocalized, cancel each other. As a result, the SD is small here and tends to zero when a properly correlated method is adopted.

The SD at the $\mathrm{CC}$ along $\mathrm{Cu}^{\prime}-\mathrm{N} 1$ and close to $\mathrm{N} 1$ is low [0.019 and 0.005 a.u. at the UB3LYP and CASSCF $(6,6)$ levels, respectively] compared with that at the facing $\mathrm{CD}$ in the VSCD of $\mathrm{Cu}^{\prime}$ along $\mathrm{Cu}^{\prime}-\mathrm{N} 1$, but still relevant, especially at the UB3LYP level. Its pattern of sources is very revealing (Fig. $8)$. The $\mathrm{N} 1$ bridging atom and the two $\mathrm{Cu}$ atoms all co-operate with their $\alpha$ effect to the positive SD at the $r p$, while $\mathrm{N}^{\prime}$ and all other $\mathrm{N}$ atoms of the pyridine ligands tend to contrast this SD accumulation with their clear $\beta$ effect. This behaviour highlights the co-operative mechanisms, acting at the $r p$, in favour or against spin delocalization from one paramagnetic to the other paramagnetic centre through the bridging N1 atom of the azido ligand. All sources of atoms along this ferromagnetic pathway concur, while those related to atoms linked through other magnetic pathways are working against. As expected, being related to the spin delocalization mechanism, the SF pattern is here essentially determined by the magnetic component. The UB3LYP and $\operatorname{CASSCF}(6,6)$ methods agree qualitatively in their description, but largely differ from a quantitative point of view. Since the UB3LYP method exaggerates spin delocalization to the ligands, it also predicts a larger positive source from N1 than from the two paramagnetic centres, while these latter dominate the CASSCF $(6,6)$ description. CASSCF $(6,6)$ also gives a much larger relative weight to the opposing effect from sources related to the atoms of the non-directly involved magnetic pathways. Once more we find that a larger spin delocalization to ligands (UB3LYP method) does not imply a relatively larger SF communication among the network of atoms involved in the spin delocalization.

In terms of an orbital language, the positive $\mathrm{SD}$ region around the bridging $\mathrm{N}$ (where its lone pair CCs are located), pointing towards the $\mathrm{Cu}^{\mathrm{II}}$ cations, may be explained in two ways. Either in terms of spin delocalization through the mixing of the $\mathrm{Cu} d_{x^{2}-y^{2}}$ orbitals and the $\pi$ gerade and $\pi$ ungerade orbitals of the azido ligands (Aebersold et al., 1998) or also in terms of a superexchange mechanism between the two $\mathrm{Cu}^{\text {II }}$
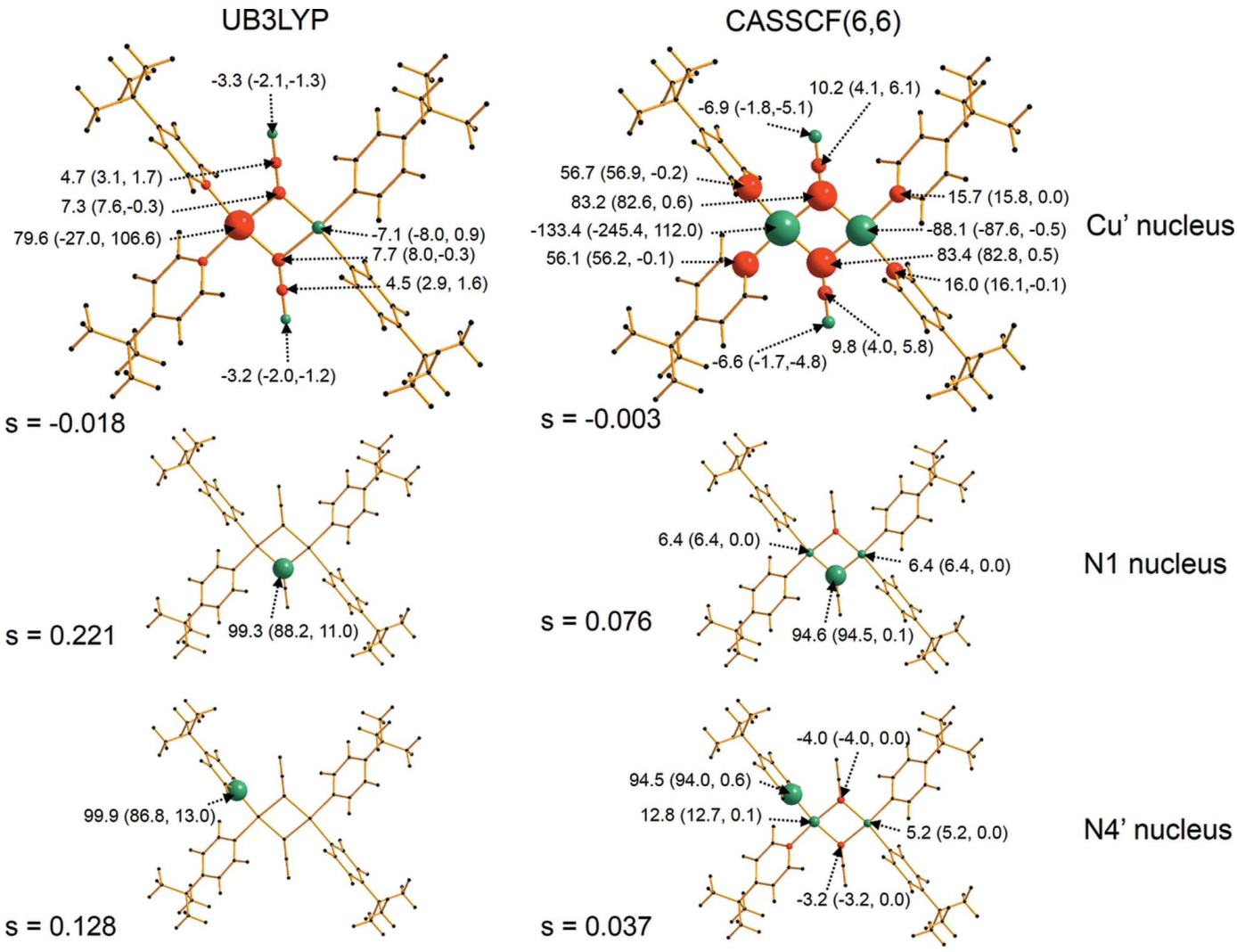

Figure 9

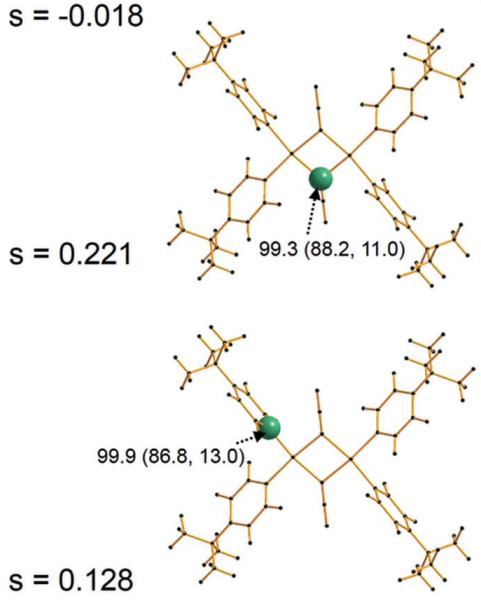

End-on FM complex: UB3LYP and CASSCF(6,6) SF percentage contributions to the $s$ (magnetic and relaxation components in parentheses) at selected nuclear positions. The $s$ values at $r p s$ are in a.u. Green (red) atomic balls denote an $\alpha(\beta)$ effect on the density at the reference point. SF percentage contributions are positive or negative whether they concur or oppose to reconstruct the corresponding density value at the indicated nucleus. 
cations and the intervening bridging $\mathrm{N} 1$ atom, when this latter subtends an angle of about $90^{\circ}$ (Elliott, 1998). In such a case, ferromagnetic coupling is favoured due to the mixing with an excited configuration having two unpaired electrons in different and orthogonal orbitals on the bridging atom, and whose associated densities are reminiscent of the shape of the positive SD region found around the bridging $\mathrm{N}$. While these are just orbital models, the SF analysis provides a rigorous picture of such orbital interaction mechanisms at the N1 CC in terms of delocalized and co-operating sources along the $\mathrm{Cu}^{\prime}-$ $\mathrm{N} 1-\mathrm{Cu}$ pathway.

Fig. 8 also shows the atomic SF spin density pattern for an $r p$ taken at the $\mathrm{Cu}^{\prime}-\mathrm{N} 1 \mathrm{BCP}$. SD is here small and positive [0.002 and 0.007 a.u. at the UB3LYP and CASSCF $(6,6)$ levels, respectively]. It is over-determined by the magnetic (positive) and opposed by the relaxation (negative) SD components, the former essentially due to the source from the $\mathrm{Cu}^{\prime}$ atom and the latter largely due to the sources from the bridging and central atoms of the azido ligands (Figs. 5 and 6 ). The magnetic source from these $\mathrm{N}$ atoms also yields a $\beta$ effect at the BCP. Therefore, $\mathrm{SF}$ contributions from the bonded $\mathrm{Cu}^{\prime}$ and $\mathrm{N} 1$ atoms are clearly opposite in sign, i.e. $\alpha$ for $\mathrm{Cu}^{\prime}$ and $\beta$ for N1. At the UB3LYP level, these SF contributions are respectively four and two times larger in magnitude than the SD they contribute to reconstruct [two and half this magnitude and the same magnitude of the SD at the CASSCF $(6,6)$ level, respectively]. Opposing sources from the bonded atoms imply that a spin polarization mechanism operates in the bonding region at the $\mathrm{BCP}$; however, with the sources being still essentially due to the magnetic component, especially at the $\operatorname{CASSCF}(6,6)$ level. One notices that there are large contributions from all the $\mathrm{N}$ of the azido ligands and also from the other $\mathrm{Cu}$ atom, which, differently from $\mathrm{N}^{\prime}$, co-operates with $\mathrm{Cu}^{\prime}$ in determining the positive $\mathrm{SD}$ at the $\mathrm{Cu}^{\prime}-\mathrm{N}$ BCP.

Fig. 9 shows the SF spin density reconstruction at the $\mathrm{Cu}^{\prime}$, $\mathrm{N} 1$ and $\mathrm{N}^{\prime}$ nuclei. At the $\mathrm{N} 1$ nucleus, the SD is very large
[0.221 and 0.076 a.u. at the UB3LYP and CASSCF(6,6) levels, respectively] and essentially due to the magnetic component at the CASSCF $(6,6)$ level, while at the UB3LYP level also the relaxation density co-operates. The three-times-larger SD at the UB3LYP level results from the observed exaggeration of spin delocalization on ligands by this model. However, while at the UB3LYP level the SD at the N1 nucleus is almost only due to the source from its own atom, the $\operatorname{CASSCF}(6,6)$ model highlights the role of the co-operating $\alpha$ sources from the two $\mathrm{Cu}$ atoms, able to cause $13 \%$ of the SD at the N1 nucleus. Again, the UB3LYP model exaggerates the amount of spin delocalization on the ligands and underestimates spin connection or communication mechanisms. Similar observations may be made for the reconstruction of the SD at the $\mathrm{N}^{\prime}$ nucleus. At the CASSCF $(6,6)$ level, both $\mathrm{Cu}^{\prime}(12.8 \%)$ and the remote $\mathrm{Cu}$ atom (5.2\%) co-operate with the $\mathrm{N}^{\prime}$ atom $(94.5 \%)$ in determining a significant $\mathrm{SD}$ (0.037 a.u.) on the $\mathrm{N} 4$ ' nucleus, while such a mechanism is totally absent at the UB3LYP level, despite a three-times-larger SD at its nucleus (0.128 a.u.). SD at the $\mathrm{Cu}^{\prime}$ nucleus is negative and close to zero $(-0.003$ a.u.) at the CASSCF $(6,6)$ level, while it is six times larger in magnitude $(-0.018)$ at the UB3LYP level. The two methods predict quite different patterns of sources. The relatively large SD at the UB3LYP level is probably due to the limits of such a model.

Indeed, at the CASSCF $(6,6)$ level, the marginal negative $\mathrm{SD}$ at $\mathrm{Cu}^{\prime}$ is due to an almost perfect balance between large opposing $\alpha$ effects from the two $\mathrm{Cu}$ atoms and negative delocalized sources from the other atoms. Looking in more detail, the sources of all atoms are almost due only to the magnetic component of the SD, except for $\mathrm{Cu}^{\prime}$, for which the relaxation component also plays a major role.

Fig. S3 of the supporting information reports SF patterns for rps along $\mathrm{Cu}^{\prime}-\mathrm{N}^{\prime}$ corresponding to those along $\mathrm{Cu}^{\prime}-\mathrm{N} 1$ shown in Fig. 8. These patterns all closely resemble those already discussed for $r p s$ along $\mathrm{Cu}^{\prime}-\mathrm{N} 1$ and may be similarly interpreted.
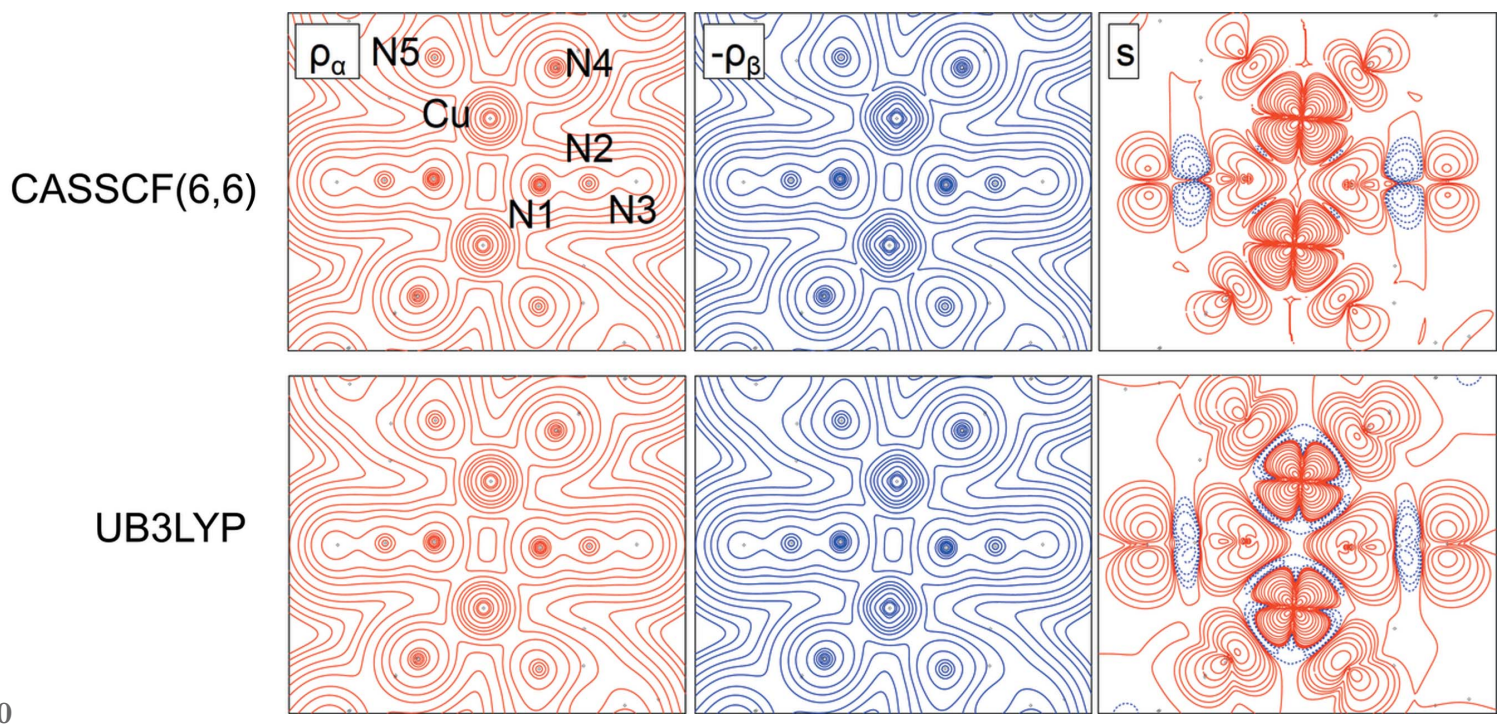

Figure 10

End-on (EO) FM complex: CASSCF(6,6) and UB3LYP contour plots of spin-resolved, $\rho_{\alpha},-\rho_{\beta}$ electron densities and of electron spin density $s=\rho_{\alpha}-\rho_{\beta}$, in the least-squares plane of the four ligand $\mathrm{N}$ atoms around each $\mathrm{Cu}^{\mathrm{II}}$. The same colour codes and isovalue contours occur as in Fig. 1. 
5.5. Comparing spin-resolved components versus spin densities: a test for the model quality

We have thus far analysed spin densities and their Laplacian, but not their spin-resolved $\rho_{\alpha}$ and $\rho_{\beta}$ components, as performed by Deutsch et al. (2014), using a split-spin multipolar model to jointly refine X-ray and PND data. For the EE complex, Deutsch et al. (2014) reported a fair agreement between the so-obtained spin-resolved components and those estimated using a UB3LYP DFT approach. A question that needs to be addressed before concluding is whether an agreement on the spin-resolved components obtained by two different approaches also ensures a similar agreement of their resulting SDs. Fig. 10 compares contour plots of $\rho_{\alpha},-\rho_{\beta}$ and of their sum, the spin density $s=\rho_{\alpha}+\left(-\rho_{\beta}\right)$, for the EO complex evaluated at the $\operatorname{CASSCF}(6,6)$ and at the UB3LYP levels. Contour plots are drawn in the least-squares plane of the four ligand $\mathrm{N}$ atoms around each $\mathrm{Cu}^{\mathrm{II}}$ (see Fig. 2). The spinresolved components appear to be similarly described by the two considered methods. In particular, $\rho_{\alpha}$ has a circular shape around the $\mathrm{Cu}$ nucleus, whereas $\rho_{\beta}$ has the rounded square shape one anticipates for an incomplete filling of the $\mathrm{Cu} d_{x^{2}-y^{2}}$ orbital, with $\mathrm{CDs}$ along the $\mathrm{Cu}^{\mathrm{II}}$-to-ligand bonds and $\mathrm{CCs}$ between adjacent CDs. In other words, the $\alpha$ electrons (almost) completely fill the five $d \alpha$-spin orbitals on $\mathrm{Cu}$, while the minority $\beta$-spin electrons incompletely fill the $\beta$-spin orbitals, leading to the discussed CDs and CCs around the $\mathrm{Cu}$ nucleus. On the other hand, noticeable qualitative differences may be observed between the SDs maps obtained at the $\operatorname{CASSCF}(6,6)$ and at the UB3LYP levels, despite the evident similarities between their spin-resolved components. As discussed earlier, a much larger spin delocalization to the ligands, along with the consequent lower SD around $\mathrm{Cu}^{\mathrm{II}}$ and the negative SD region at its boundaries is visibly manifested in the UB3LYP map. The qualitative shape of the $\alpha$ and $\beta$ components is imparted by all their electrons, the majority of them being described quite similarly in the two distributions as they fill doubly occupied orbitals. Comparison of the spin density, instead, singles out the difference between the distributions of only those few electrons that are either missing in the minority spin distribution or are described in a significantly different manner by the spin-resolved distributions. Different model approaches may significantly differ in the description of, namely, such few peculiar electrons.

A similar analysis was performed for the case of the EE complex. As shown in Fig. S4, the CASSCF $(6,6)$ and UB3LYP contour plots of spin-resolved electron densities, in the leastsquares plane of the $\mathrm{Cu}-\mathrm{O}$ bond and of the three shorter $\mathrm{Cu}-\mathrm{N}$ bonds, are almost indistinguishable, while those of the corresponding SDs largely differ. Our spin-resolved components also mostly agree (Fig. S4) with those obtained by Deutsch et al. (2014) from either the joint refinement of X-ray and PND data or the UB3LYP calculation (minor differences may be ascribed to the different choice of contour levels). Yet this does not imply similar SDs and atomic SD populations, as demonstrated here for the EO complex. Results for the EE complex, with a change of method, follow the same trends discussed for the EO system and will be illustrated in future work.

\section{Conclusions}

Magnetic interactions between metal atoms, mediated through diamagnetic ligands acting as couplers, are customarily discussed in terms of superexchange interactions and of spin delocalization and polarization mechanisms. Orbital models (Aebersold et al., 1998; Aronica et al., 2007; Dos Santos et al., 2016) and/or topological analyses of the spin density (Dos Santos et al., 2016) are used to interpret the spin density (SD) distribution and obtain further insight on such interactions and mechanisms.

In this work, we applied the Source Function (SF) tool to the analysis of the SD in an azido $\mathrm{Cu}^{\mathrm{II}}$ dinuclear complex, where magnetic interactions between the metal atoms take place through the azido ligands, coordinated in an end-on mode, and also through the external pyridine ligands. Firstly, a clear relationship between the electron-density Laplacian and the SD distribution around the metal atom has been established. Then, application of the SF tool has enabled us to highlight how the magnetic centres contribute to determine the local spin delocalization and polarization at any point of the complex and whether a given atom or group of atoms of the ligands is co-operating in favour or against a certain local spin delocalization/polarization. This SF capability provides an unprecedented atomic insight on SD distributions and on the local role played by the remote or closer atomic centres, within a cause-effect view.

Reconstruction of partial SDs by means of the Source Function has been introduced for the first time. This reconstruction enables us to visualize, simultaneously, the mechanisms and effects discussed earlier, at all points of space or in selected molecular planes, and for a specific subset of contributing atoms. When all atoms of a system are included in such a subset, one is then led back to the conventional SD analysis. Partial SDs also permit an easier interpretation of the usual SF percentage reconstructions at given reference points and provide invaluable help for an appropriate choice of such points. The ball-and-stick representations of these zerodimensional SF reconstructions, allow for a visualization of the magnetic pathways and of the specific role played by each atom along these paths. Decomposition of SF contributions in terms of a magnetic and of a relaxation component provides further insight for all kinds of adopted SF representation (zero- and two-dimensional in the present study).

SD distributions may largely depend on the way they are calculated from theoretical approaches or are derived from diffraction experiments. In the present study, the SF analysis has neatly identified the similarities and differences between SD distributions obtained from a standard unrestricted DFT method, using an exchange correlation functional of the hybrid type (UB3LYP), or from a more costly and accurate CASSCF approach. UB3LYP greatly overestimates spin delocalization to the ligands, while comparatively underestimating magnetic connection (spin transmission) among 
atoms, along the magnetic pathways. Because of the excessive spin delocalization, the UB3LYP method is forced to introduce or to overestimate spin polarization mechanisms between the paramagnetic centres and the ligands, leading to an exaggerated partial covalent nature of metal-ligand interactions which eventually dampens spin transmission. Decomposition in a magnetic and in a relaxation component provides further understanding of the origin of the observed SD discrepancies. Spin delocalization from PND data seems intermediate in its extent between that calculated at the UB3LYP and at the CASSCF levels. The SF tool, when applied to ab initio SDs and to those derived from experiment, could be of great value for a better understanding of the very origin of differences. Use of a high-quality wavefunction seems to be required when comparing theoretical and experimentally derived (PND) spin densities. Despite the very small number of normally available PND structure-factor data and despite the consequent adoption of very rigid models in their refinement, SDs derived this way appear in general superior to those obtained through a DFT UB3LYP approach and closer to the far more accurate CASSCF result. The relevance of the experimentally derived SDs is further manifest when considering the difficulty in reaching a reasonable convergence of CASSCF estimates against the number of correlated electrons even for systems of a moderate size.

We also showed that a visual, almost perfect, agreement on the spin-resolved electron densities $\left(\rho_{\alpha}\right.$ and $\left.\rho_{\beta}\right)$, obtained from different approaches, does not yet ensure a corresponding agreement between their associated SDs. Lots of fundamental details and significant differences may indeed emerge from their direct comparison.

\section{Acknowledgements}

One of us (CG) wishes to warmly thank Professor Philip Coppens for his continuous interest, precious support and great mutual scientific and human discussions. One of us (LLP) thanks the Italian supercomputing centre CINECA for provision of computational time through the CINECA-Unimi convention. The three of the authors thank Dr Ahmed Muhammed Orlando and Pietro Colombo for invaluable help.

\section{Funding information}

The following funding is acknowledged: Danmarks Grundforskningsfond (award No. DNRF93).

\section{References}

Aebersold, M., Gillon, B., Plantevin, O., Pardi, L., Kahn, O., Bergerat, P., von Seggern, I., Tuczek, F., Öhrström, L., Grand, A. \& LelièvreBerna, E. (1998). J. Am. Chem. Soc. 120, 5238-5245.

Arfken, G. (1985). Mathematical Methods for Physicists. Orlando: Academic Press.

Aronica, C., Jeanneau, E., El Moll, H., Luneau, D., Gillon, B., Goujon, A., Cousson, A., Carvajal, M. A. \& Robert, V. (2007). Chem. Eur. J. 13, 3666-3674.

Bader, R. F. W. (1990). Atoms in Molecules: A Quantum Theory. Oxford University Press.

Bader, R. F. W. \& Gatti, C. (1998). Chem. Phys. Lett. 287, 233-238.
Bianchi, R., Gatti, C., Adovasio, V. \& Nardelli, M. (1996). Acta Cryst. B52, 471-478.

Biegler-König, F. W., Bader, R. F. W. \& Tang, T.-H. (1982). J. Comput. Chem. 3, 317-328.

Boguslawski, K., Jacob, C. R. \& Reiher, M. (2011). J. Chem. Theory Comput. 7, 2740-2752.

Boguslawski, K., Marti, K. H., Legeza, O. \& Reiher, M. (2012). J. Chem. Theory Comput. 8, 1970-1982.

Conradie, J. \& Ghosh, A. (2007). J. Phys. Chem. B, 111, 12621-12624.

Coppens, P. (1997). X-ray Charge Densities and Chemical Bonding. Oxford University Press.

Coppens, P. (2009). Angew. Chem. Int. Ed. 48, 4280-4281.

Coppens, P. (2015). Phys. Scr. 90, 058001.

Deutsch, M., Claiser, N., Pillet, S., Chumakov, Y., Becker, P., Gillet, J.-M., Gillon, B., Lecomte, C. \& Souhassou, M. (2012). Acta Cryst. A68, 675-686.

Deutsch, M., Gillon, B., Claiser, N., Gillet, J.-M., Lecomte, C. \& Souhassou, M. (2014). IUCrJ, 1, 194-199.

Dos Santos, L. H. R., Lanza, A., Barton, A. M., Brambleby, J., Blackmore, W. J. A., Goddard, P. A., Xiao, F., Williams, R. C., Lancaster, T., Pratt, F. L., Blundell, S. J., Singleton, J., Manson, J. L. \& Macchi, P. (2016). J. Am. Chem. Soc. 138, 2280-2291.

Elliott, S. R. (1998). The Physics and Chemistry of Solids. Chichester: John Wiley and Sons.

Farrugia, L. J., Evans, C. \& Tegel, M. (2006). J. Phys. Chem. A, 110, 7952-7961.

Frisch, M. J. et al. (2009). GAUSSIAN09, Revision A.1. Gaussian, Inc., Wallingford, CT, USA.

Gatti, C. (2005). Z. Kristallogr. 220, 399-457.

Gatti, C. (2012). Struct. Bonding, 147, 193-286.

Gatti, C. (2013). Phys. Scr. 87, 048102.

Gatti, C., Cargnoni, F. \& Bertini, L. (2003). J. Comput. Chem. 24, 422436.

Gatti, C. \& Lasi, D. (2007). Faraday Discuss. 135, 55-78.

Gatti, C. \& Macchi, P. (2012). Modern Charge Density Analysis, edited by C. Gatti and P. Macchi, ch. 1, pp. 1-78. Dordrecht, Heidelberg, London, New York: Springer.

Gatti, C., Orlando, A. M. \& Lo Presti, L. (2015). Chem. Sci. 6, 38453852.

Gatti, C., Orlando, A. M., Monza, E. \& Lo Presti, L. (2016). Applications of Topological Methods in Molecular Chemistry, edited by R. Chauvin, C. Lepetit, B. Silvi \& E. Alikhani, Vol. 22, ch. 5, pp. 101-129. Switzerland: Springer International Publishing.

Gatti, C., Saleh, G. \& Lo Presti, L. (2016). Acta Cryst. B72, 180193.

Gillet, J.-M. (2007). Acta Cryst. A63, 234-238.

Gillet, J.-M., Becker, P. J. \& Cortona, P. (2001). Phys. Rev. B, 63, $235115,1-7$.

Gillet, J.-M. \& Koritsanszky, T. (2012). Modern Charge Density Analysis, edited by C. Gatti \& P. Macchi, ch. 5, pp. 181-211. Dordrecht, Heidelberg, London, New York: Springer.

Gillon, B. \& Becker, P. (2012). Modern Charge Density Analysis, edited by C. Gatti \& P. Macchi, ch. 8, pp. 277-302. Dordrecht, Heidelberg, London, New York: Springer.

Gillon, B., Sangregorio, C., Caneschi, A., Gatteschi, D., Sessoli, R., Ressouche, E. \& Pontillon, Y. (2007). Inorg. Chim. Acta, 360, 38023806.

Hansen, N. K. \& Coppens, P. (1978). Acta Cryst. A34, 909-921.

Herzberg, G. (1966). Molecular Spectra and Molecular Structure. III. Electronic Spectra and Electronic Structure of Polyatomic Molecules. New York: Van Nostrand Reinhold Company.

Koritsanszky, T. S. \& Coppens, P. (2001). Chem. Rev. 101, 15831627.

Lo Presti, L. \& Destro, R. (2008). J. Chem. Phys. 128, 044710.

Macchi, P., Gillet, J.-M., Taulelle, F., Campo, J., Claiser, N. \& Lecomte, C. (2015). IUCrJ, 2, 441-451.

Macchi, P. \& Sironi, A. (2003). Coord. Chem. Rev. 238-239, 383412. 
Peintinger, M. F., Oliveira, D. V. \& Bredow, T. (2013). J. Comput. Chem. 34, 451-459.

Pendás, A. M., Francisco, E., Blanco, M. A. \& Gatti, C. (2007). Chem. Eur. J. 12, 9362-9371.

Ponec, R. \& Gatti, C. (2009). Inorg. Chem. 48, 11024-11031.

Putz, H. \& Brandenburg, K. (1997-2012). DIAMOND. Crystal Impact GbR, Bonn, Germany.
Stewart, R. F., Bentley, J. \& Goodman, B. (1975). J. Chem. Phys. 63, 3786-3793.

Volkov, A. V., Macchi, P., Farrugia, L. J., Gatti, C., Mallinson, P., Richter, T. \& Koritsanszky, T. (2006). XD2006. University at Buffalo, State University of New York, NY, USA; University of Milano, Italy; University of Glasgow, UK; CNR-ISTM, Milano, Italy; Middle Tennessee State University, TN, USA. 